\title{
PSEUDO-STATIC PASSIVE RESPONSE OF RETAINING WALL SUPPORTING c-Ф BACKFILL
}

\author{
Sima Ghosh ${ }^{1}$ and Sumen Deb ${ }^{2}$ \\ ${ }^{1}$ Assistant Professor, ${ }^{2}$ PG student, Civil Engineering Department, National Institute of Technology Agartala, Tripura, \\ India, sima.civil@nita.ac.in,d_sumen@yahoo.co.in
}

\begin{abstract}
Passive resistance is a significantly important factor for successful design and performance of various structures like anchors, bulkheads, retaining walls etc. Several analytical methods have been introduced time to time to predict the passive resistance for retaining walls supporting soil as the backfill. Most of these methods for the analysis are based on linear failure criterion. Whereas; experimental investigations, theoretical analysis and failed structures have indicated that the rupture surface is supposed to be nonlinear for the most practical environment. Thus, the assumption of planar sliding surface is supposed to underestimate the lateral earth pressure on the active side, which may make retaining walls unsatisfactorily designed at the passive side for support depending on earth pressures. For this reason, the nonlinear analyses were introduced in the earth pressure theories. The methodologies for nonlinear analysis under seismic loading conditions are mostly based on the assumption of log spiral failure surface. Eminent researchers have predicted the failure surface to be a combination of log spiral and straight line. In this paper an effort has been made to derive the analytical expression of passive earth pressure coefficient on the retaining wall from the c- $\Phi$ backfill subjected to both horizontal and vertical seismic coefficients. The solution has been carried out by using Horizontal Slices Method (HSM) and limit equilibrium principles to generate a non-linear failure surface. Pseudo-static approach has been used to determine the seismic passive earth pressure. Generalized equation has been developed to find the solution. Results have been prepared in tabular form considering variation of parameters. The results have duly been compared with previous studies to justify the present analysis. Detailed parametric study has been made for the variation of different parameters like angle of internal friction ( $\Phi)$, angle of wall friction $(\delta)$, wall inclination angle $(\alpha)$, Horizontal and vertical seismic coefficients ( $k_{h}$ and $\left.k_{v}\right)$, cohesion $(c)$, adhesion $\left(c_{a}\right)$ and height of retaining wall $(H)$.
\end{abstract}

Index Terms:- Pseudo-static, seismic passive earth pressure, c- $\Phi$ backfill, rigid retaining wall, Wall inclination, nonlinear failure surface.

\section{INTRODUCTION}

The passive resistance refers to a condition which enables the resistance of a mass of soil against the movement of the structure. The concept is very important for the stability of various structures like anchors, bulk heads and also for bearing capacity of foundation etc. In common practice, the total static passive earth pressure or force from soil backfills is calculated using the methods based on Rankine's (1857) or Coulomb's (1776) analytical expressions. But, retaining walls are exposed to the extreme unfavorable effects of earthquakes and its strong dynamic waves. The very first expressions from Okabe (1926) and Mononobe-Matsuo (1929) analysis has provided the solution for dynamic earth pressure considering the wall backfilled by $\Phi$ nature of soil. They extended Coulomb wedge (1776) theory for evaluating dynamic earth pressure by incorporating the seismic acceleration as inertia forces. Kumar and Subba Rao (1997) adopted a method of slices to predict the passive earth pressure co-efficient. Ghosh and Sengupta (2012) and Sharma and Ghosh (2012) have suggested essential solutions for $\mathrm{c}-\Phi$ nature of backfill under seismic loading conditions. These analyses are mainly based on linear nature of failure surfaces. Terzaghi (1943) has given a solution by considering $\log$ spiral failure for the analysis of lateral earth pressure to show the nonlinearity of failure surface. The log spiral method was adopted by Kumar (2001), where the passive earth pressure co-efficient for an inclined retaining wall has been computed by taking the failure surface as a combination of a logarithmic spiral and a straight line. Subba Rao and Choudhury (2005) analyzed the seismic earth pressure in soils using the limit equilibrium method based on pseudo-static approach and considering the effects of cohesion (c) in soils. Whereas, Azad et. al. (2008) and Ghanbari and Ahmadabadi (2010) have given the solution by considering the Horizontal Slices Method with linear kind of failure surface. The passive resistance from cohesionless ( $\Phi$ ) backfills has been analyzed by Choudhury and Nimbalkar (2005) considering the concept of phase difference due to finite shear wave propagation using pseudo-dynamic methods. From the earlier studies, it reveals that specially, in case of passive condition, non-linear failure surface generates more acceptable solution in comparison to linear failure surface 
analyses. Therefore, in this study, an attempt has been made to generate a non-linear failure surface. To do this analysis, Horizontal Slice Method is used.

\section{ANALYTICAL SOLUTION}

For the analysis, let us consider a retaining wall inclined at an angle, $\alpha$ with the vertical as shown in Fig.1. The wall of height, $H$ retains a horizontal $c-\Phi$ backfill and the failure surface of the retaining wall is considered to be non-linear as shown in the figure. The failure surface makes the angles of $\theta_{\mathrm{n}}$ with the vertical at bottom and $\theta_{1}$ with the vertical at the top as shown in Figure.

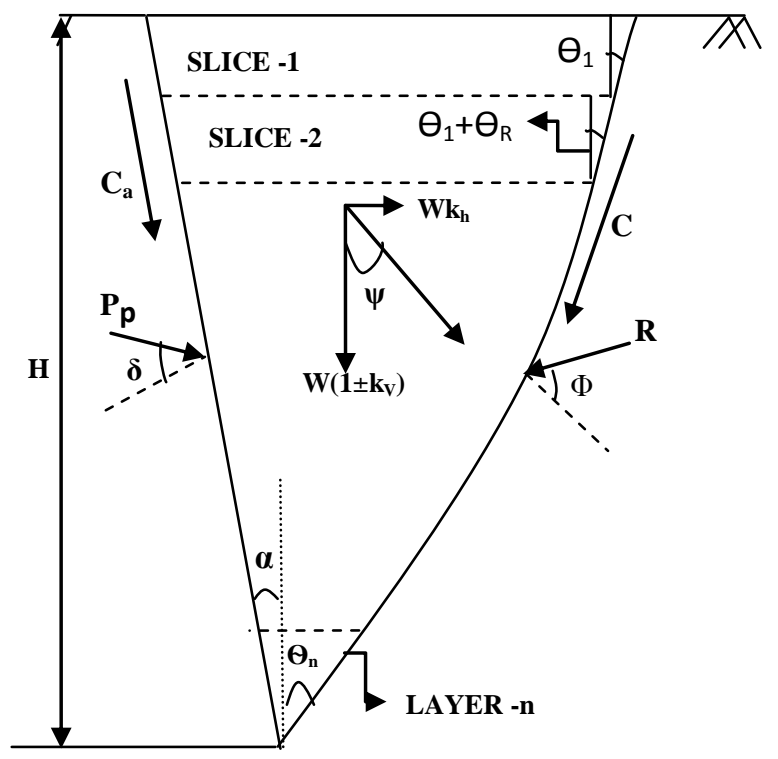

Fig.1 Battered face retaining wall under passive pseudo-static state of equilibrium

The failure wedge is divided into ' $n$ ' number of slices with equal thickness of $\Delta H$ as shown in Fig.2. The assumptions for various parameters related to slices have been detailed in Fig.2. The rate of change of inclination of failure surface with the vertical $\left(\theta_{1}\right.$ to $\left.\theta_{\mathrm{n}}\right)$ has been assumed as $\theta_{\mathrm{R}}=\left\{\left(\theta_{1} \sim \theta_{\mathrm{n}}\right) /(\mathrm{n}\right.$ 1)\}. Free Body Diagram of retaining wall-backfill system under passive pseudo-static state of equilibrium has been elaborated in Fig.2.

The forces acting on the wall has been calculated by considering the following parameters:

$\mathrm{H}_{\mathrm{i}-1}, \mathrm{H}_{\mathrm{i}}=$ Horizontal shear acting on the top and bottom of the $i^{\text {th }}$ slice.

$\mathrm{W}_{\mathrm{i}}=$ Weight of the failure wedge of $\mathrm{i}^{\mathrm{th}}$ slice.

$\mathrm{V}_{\mathrm{i}-1}, \mathrm{~V}_{\mathrm{i}}=$ Vertical load (UDL) on top and bottom of $\mathrm{i}^{\text {th }}$ slice.

$\Phi=$ The angle of internal friction of soil.

$\mathrm{P}_{\mathrm{i}}=$ Passive earth pressure on $\mathrm{i}^{\text {th }}$ slice.

$\mathrm{R}_{\mathrm{i}}=$ The reaction of the retained soil on $\mathrm{i}^{\text {th }}$ slice.

$\delta=$ The angle of wall friction.
$\mathrm{C}=$ Cohesion acting on the failure surface.

$\mathrm{C}_{\mathrm{a}}=$ Adhesion acting on the wall surface.

$\mathrm{k}_{\mathrm{h}}=$ Horizontal seismic coefficient.

$\mathrm{k}_{\mathrm{v}}=$ Vertical seismic coefficient.

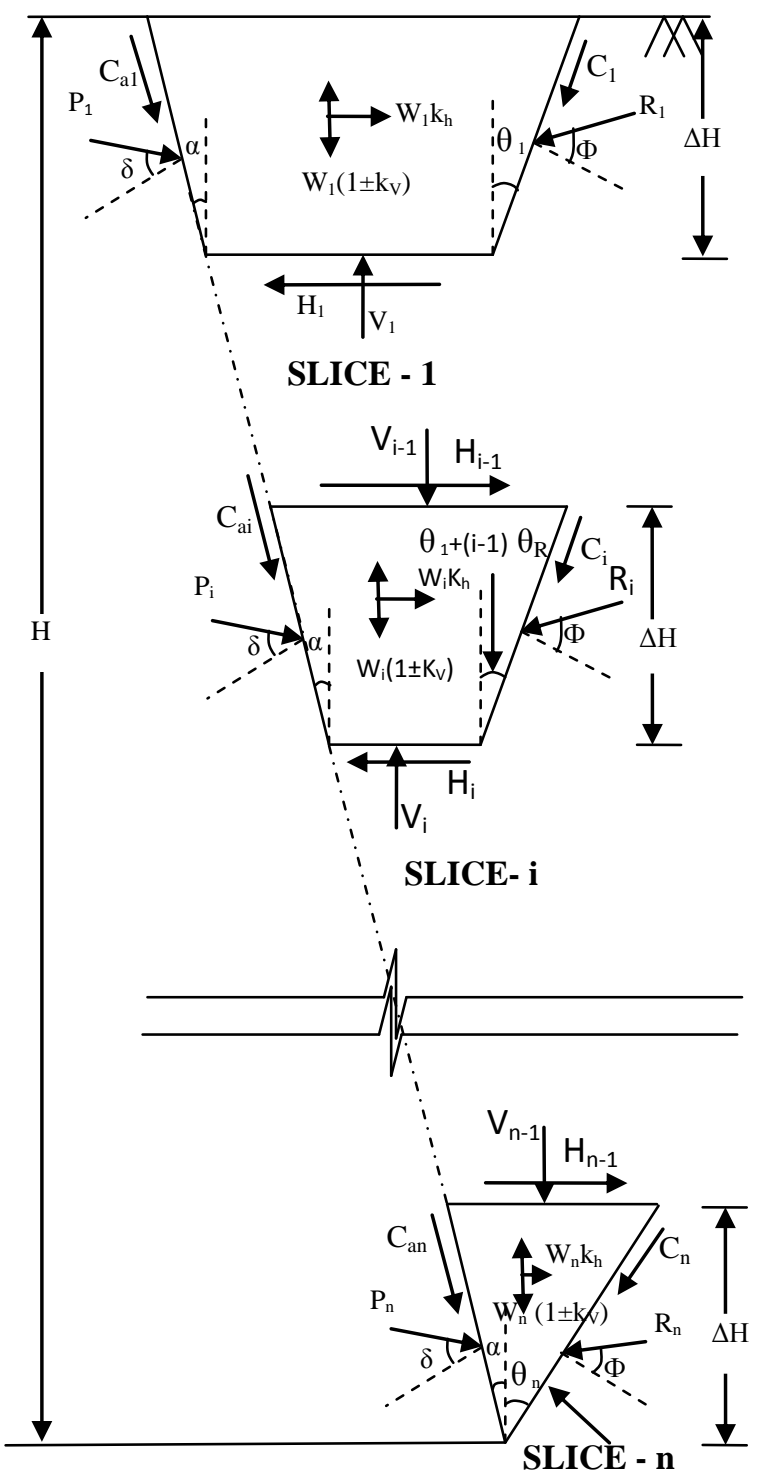

Fig.2: Showing the forces acting on wedge slices during passive state of equilibrium $(1<\mathrm{i}<\mathrm{n})$

\section{DERIVATION OF FORMULATIONS CONSIDERING PASSIVE STATE OF EQUILIBRIUM}

Applying the force equilibrium conditions for $1^{\text {st }}$ slice from Fig.2, we can solve the equations in the following pattern:

$\sum H=0$ 
$P_{1} \cos (\delta-\alpha)=R_{1} \cos \left(\Phi-\theta_{1}\right)-\frac{1}{2} \gamma(\Delta \mathrm{H})^{2}\left(\tan \alpha+\tan \theta_{1}\right) k_{h}$

$+\frac{c(\Delta \mathrm{H}) \sin \theta_{1}}{\cos \left(\theta_{1}+(i-1) \theta_{R}\right)}-\frac{c_{a}(\Delta \mathrm{H}) \sin \alpha}{\cos \alpha}$

$+\gamma(\Delta \mathrm{H})^{2}\left\{\tan \Phi\left(\tan \left(\theta_{1}+\theta_{r}\right)+\right.\right.$

$+(n-1) \tan \alpha-c(\Delta \mathrm{H})(\tan \alpha+\tan \theta)\}$

$\sum V=0$

$P_{1} \sin (\delta-\alpha)=-R_{1} \sin \left(\Phi-\theta_{1}\right)$

$-\frac{1}{2} \gamma(\Delta \mathrm{H})^{2}\left(\tan \alpha+\tan \theta_{1}\right)\left(1 \pm k_{v}\right)$

$-\frac{c(\Delta \mathrm{H}) \cos \theta_{1}}{\cos \theta_{1}}-\frac{c_{a}(\Delta \mathrm{H}) \cos \alpha}{\cos \alpha}$

Solving these equations ( 1 and 2), we get,

$$
P_{P 1}=\frac{\left[\begin{array}{l}
(\Delta \mathrm{H})^{2}\left(1 \pm k_{v}\right)\left[\left\{\left(\tan \alpha+\tan \theta_{1}\right)\right.\right. \\
\left(-\tan \psi \sin \left(\Phi-\theta_{1}\right)-\cos \left(\Phi-\theta_{1}\right)\right\} \\
+\frac{N_{s} \cos \Phi}{\cos \theta_{1}}+\frac{M_{s} \cos \left(\Phi-\alpha-\theta_{1}\right)}{\cos \alpha} \\
+\left(\frac { 2 \operatorname { t a n } \Phi } { ( 1 \pm k _ { v } ) } \left\{\left(\tan \left(\theta_{1}+\theta_{R}\right)+\ldots \ldots \ldots \ldots\right.\right.\right. \\
\cdots \ldots \ldots \ldots . .+\tan \left(\theta_{1}+(n-1) \theta_{R}\right) \\
\left.+(n-1) \tan \alpha-N_{s}(\tan \alpha+\tan \theta) \sin \left(\Phi-\theta_{1}\right\}\right]
\end{array}\right]}{\sin \left(\alpha+\theta_{1}-\Phi-\delta\right)}
$$

Where, $\tan \psi=\frac{k_{h}}{1 \pm k_{v}}$

In the present study $\mathrm{N}_{\mathrm{s}}$ and $\mathrm{M}_{\mathrm{s}}$ Values have been introduced for the analysis of slices, where

$$
\begin{aligned}
& \mathrm{N}_{\mathrm{s}}=(\mathrm{H} / \Delta \mathrm{H}) \mathrm{N}_{\mathrm{c}} \\
& \mathrm{M}_{\mathrm{s}}=(\mathrm{H} / \Delta \mathrm{H}) \mathrm{M}_{\mathrm{c}}
\end{aligned}
$$

Where,

$$
\begin{aligned}
& \mathrm{N}_{\mathrm{c}}=(2 \mathrm{c} / \gamma \mathrm{H}) \\
& \mathrm{M}_{\mathrm{c}}=\left(2 \mathrm{c}_{\mathrm{a}} / \gamma \mathrm{H}\right)
\end{aligned}
$$

$$
\frac{\gamma}{P_{P 2}}=\frac{\left[\begin{array}{l}
(\Delta \mathrm{H})^{2}\left(1 \pm k_{v}\right)\left[\left\{3\left(\tan \alpha+\tan \left(\theta_{1}+\theta_{R}\right)\right)\right.\right. \\
\left(-\tan \psi \sin \left(\Phi-\theta_{1}-\theta_{R}\right)\right. \\
\left.\left.-\cos \left(\Phi-\theta_{1}-\theta_{R}\right)\right)\right\} \\
+\frac{N_{s} \cos \Phi}{\cos \left(\theta_{1}+\theta_{r}\right)}+\frac{M_{s} \cos \left(\Phi-\alpha-\theta_{1}-\theta_{R}\right)}{\cos \alpha} \\
+\left(\frac { 2 \operatorname { t a n } \Phi } { ( 1 \pm k _ { v } ) } \left\{\left(\tan \left(\theta_{1}+2 \theta_{R}\right)\right.\right.\right. \\
+\ldots \ldots \ldots \ldots \ldots \ldots \ldots \ldots+\tan \left(\theta_{1}+(n-1) \theta_{R}\right) \\
\left.+(n-2) \tan \alpha-\left(\tan \left(\theta_{1}+\theta_{R}\right)+\tan \alpha\right)\right) \\
\left.-N_{s}\left(\tan \alpha+\tan \left(\theta_{1}+\theta_{R}\right)\right)\right) \\
\left.\left.\sin \left(\Phi-\theta_{1}-\theta_{R}\right)\right\}\right]
\end{array}\right.}{\sin \left(\alpha+\theta_{1}+\theta_{R}-\Phi-\delta\right)}
$$

Similarly, for $\mathrm{n}^{\text {th }}$ slice, we get,

$$
\begin{aligned}
& \sum H=0 \\
& P_{n} \cos (\delta-\alpha)=R_{n} \cos \left(\Phi-\theta_{1}-(n-1) \theta_{R}\right) \\
& -\left(n-\frac{1}{2}\right) \gamma(\Delta \mathrm{H})^{2}\left\{\tan \alpha+\tan \left(\theta_{1}+(n-1) \theta_{r}\right)\right\} k_{h} \\
& +\frac{c(\Delta \mathrm{H}) \sin \left(\theta_{1}+(n-1) \theta_{R}\right)}{\cos \left(\theta_{1}+(n-1) \theta_{R}\right)}-\frac{c_{a}(\Delta \mathrm{H}) \sin \alpha}{\cos \alpha}
\end{aligned}
$$$$
+\gamma(\Delta \mathrm{H})^{2}\left[\begin{array}{l}
\left\{\left[\sum_{m=i}^{n-1} \tan \Phi\left(\tan \left(\theta_{1}+m \theta_{r}\right)\right]\right.\right. \\
-(n-1)\left(\tan \left(\theta_{1}+(n-1) \theta_{R}\right)+\tan \alpha\right) \\
\left.-c(\Delta \mathrm{H})\left(\tan \alpha+\tan \left(\theta_{1}+(n-1) \theta_{R}\right)\right)\right\}
\end{array}\right]
$$

$$
\sum V=0
$$

$$
\begin{aligned}
& P_{n} \sin (\delta-\alpha)=-R_{n} \sin \left(\Phi-\theta_{1}-(n-1) \theta_{R}\right) \\
& -\left(n-\frac{1}{2}\right) \gamma(\Delta \mathrm{H})^{2}\left(\tan \alpha+\tan \left(\theta_{1}\right.\right. \\
& \left.\left.+(n-1) \theta_{R}\right)\right)\left(1 \pm k_{v}\right) \\
& -\frac{c(\Delta \mathrm{H}) \cos \left(\theta_{1}+(n-1) \theta_{R}\right)}{\cos \left(\theta_{1}+(n-1) \theta_{R}\right)}-\frac{c_{a}(\Delta \mathrm{H}) \cos \alpha}{\cos \alpha}
\end{aligned}
$$




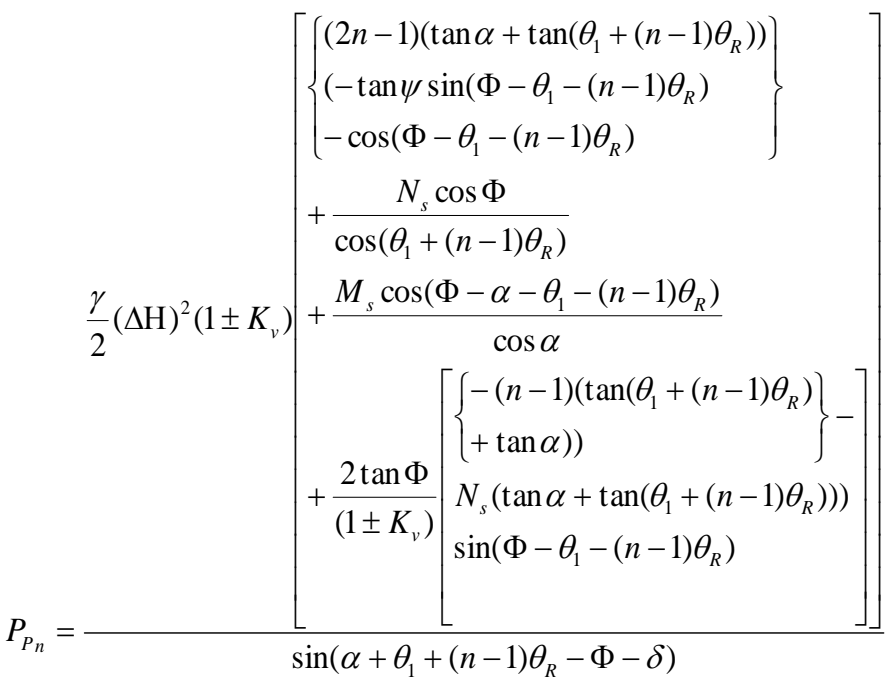

Thus, Total passive resistance of the backfill can be stated as,

$$
\mathrm{P}_{\mathrm{p}}=\mathrm{P}_{\mathrm{p} 1}+\mathrm{P}_{\mathrm{p} 2}+\mathrm{P}_{\mathrm{p} 3}+\ldots \ldots \ldots+\mathrm{P}_{\mathrm{pn}}
$$

Now the generalized equation for $\mathrm{i}^{\text {th }}$ slice can be sorted out as follows,

$\sum H=0$;

$P_{i} \cos (\delta-\alpha)=R_{i} \cos \left(\Phi-\theta_{1}-(i-1) \theta_{R}\right)$

$-\left(i-\frac{1}{2}\right) \gamma(\Delta \mathrm{H})^{2}\left\{\tan \alpha+\tan \left(\theta_{1}+(i-1) \theta_{r}\right)\right\} k_{h}$

$+\frac{c(\Delta \mathrm{H}) \sin \left(\theta_{1}+(i-1) \theta_{R}\right)}{\cos \left(\theta_{1}+(i-1) \theta_{R}\right)}$

$-\frac{c_{a}(\Delta \mathrm{H}) \sin \alpha}{\cos \alpha}+\gamma(\Delta \mathrm{H})^{2}\left[\begin{array}{l}\left\{\sum_{m=i}^{n-1} \tan \Phi\left(\tan \left(\theta_{1}+m \theta_{r}\right)+\right.\right. \\ (n-i) \tan \alpha-(i-1) \\ \left.\left(\tan \left(\theta_{1}+(i-1) \theta_{R}\right)+\tan \alpha\right)\right) \\ -c(\Delta \mathrm{H})(\tan \alpha \\ \left.\left.+\tan \left(\theta_{1}+(i-1) \theta_{R}\right)\right)\right\}\end{array}\right]$

$$
\begin{aligned}
& P_{i} \sin (\delta-\alpha)=-R_{i} \sin \left(\Phi-\theta_{1}-(i-1) \theta_{R}\right) \\
& -\left(i-\frac{1}{2}\right) \gamma(\Delta \mathrm{H})^{2}\left(\tan \alpha+\tan \left(\theta_{1}\right.\right. \\
& \left.\left.+(i-1) \theta_{R}\right)\right)\left(1 \pm k_{v}\right)-\frac{c(\Delta \mathrm{H}) \cos \left(\theta_{1}+(i-1) \theta_{R}\right)}{\cos \left(\theta_{1}+(i-1) \theta_{R}\right)} \\
& -\frac{c_{a}(\Delta \mathrm{H}) \cos \alpha}{\cos \alpha}
\end{aligned}
$$

Solving the above equations (14 and 15), the generalized equations for $\mathrm{i}^{\text {th }}$ slice can be formulated as follows:

$$
P_{P i}=\frac{\gamma}{2}(\Delta \mathrm{H})^{2}\left(1 \pm k_{v}\right)\left[\begin{array}{l}
(2 i-1)\left(\tan \alpha+\tan \left(\theta_{1}\right.\right. \\
\left.\left.+(i-1) \theta_{R}\right)\right) \\
\left(-\tan \psi \sin \left(\Phi-\theta_{1}-(i-1) \theta_{R}\right)\right. \\
\left.-\cos \left(\Phi-\theta_{1}-(i-1) \theta_{R}\right)\right)
\end{array}\right]
$$

The condition to use Eqn. 16 is at, $\mathrm{i}=\mathrm{n}$, we have to take,

$$
\tan \left(\theta_{1}+m \theta_{r}\right)=0
$$

From all the above equations, the passive earth pressure coefficient can be simplified as,

$$
k_{p}=\frac{\sum_{i=1}^{n} P_{p i}}{\frac{\gamma H^{2}}{2}}
$$




\section{RESULTS AND DISCUSSIONS}

On optimization of $\mathrm{k}_{\mathrm{p}}$ with respect to $\theta_{1}$ and $\theta_{\mathrm{n}}$, we get the seismic passive earth pressure co-efficient which is denoted here as $K_{p}$. Lists of values obtained on optimization have been presented in tabulated form herewith (Table-1 to Table-8).

Table-1: Passive earth pressure coefficients $\left(\mathrm{K}_{\mathrm{p}}\right)$ for $\mathrm{N}_{\mathrm{c}}=0.1$, $\mathrm{k}_{\mathrm{h}}=0.1$

\begin{tabular}{|c|c|c|c|c|c|c|c|c|}
\hline \multirow{2}{*}{$\Phi$} & \multirow{2}{*}{$\boldsymbol{\delta}$} & \multirow{2}{*}{$\mathbf{M}_{\mathbf{c}}$} & \multicolumn{3}{|c|}{$\mathbf{k}_{\mathrm{v}}=\mathbf{0}$} & \multicolumn{3}{|c|}{$k_{v}=k_{h} / 2$} \\
\hline & & & $\begin{array}{l}\alpha=- \\
20^{\circ}\end{array}$ & $\alpha=0^{\circ}$ & $\begin{array}{l}\alpha= \\
+20^{\circ}\end{array}$ & $\begin{array}{l}\boldsymbol{\alpha}=- \\
\mathbf{2 0}^{\circ}\end{array}$ & $\alpha=0^{\circ}$ & $\begin{array}{l}\alpha= \\
+20^{\circ}\end{array}$ \\
\hline \multirow{9}{*}{20} & \multirow{3}{*}{ 0 } & 0 & 2.813 & 2.000 & 1.709 & 2.651 & 1.898 & 1.617 \\
\hline & & $\mathrm{N}_{\mathrm{c}} / 2$ & 2.905 & 2.063 & 1.746 & 2.738 & 1.951 & 1.648 \\
\hline & & $\mathbf{N}_{\mathbf{c}}$ & 2.995 & 2.117 & 1.796 & 2.823 & 2.001 & 1.691 \\
\hline & \multirow{3}{*}{$\Phi / 2$} & $\overline{0}$ & 3.960 & 2.479 & 1.984 & 3.72 & 2.34 & 1.875 \\
\hline & & $\mathrm{N}_{\mathrm{c}} / 2$ & 4.064 & 2.533 & 2.019 & 3.818 & 2.391 & 1.908 \\
\hline & & $\mathbf{N}_{\mathrm{c}}$ & 4.168 & 2.585 & 2.052 & 3.916 & 2.44 & 1.939 \\
\hline & \multirow{3}{*}{$\Phi$} & 0 & 6.147 & 3.177 & 2.319 & 5.748 & 2.991 & 2.19 \\
\hline & & $\mathbf{N}_{\mathrm{c}} / 2$ & 6.276 & 3.233 & 2.347 & 5.872 & 3.044 & 2.217 \\
\hline & & $\mathbf{N}_{\mathbf{c}}$ & 6.404 & 3.288 & 2.375 & 5.993 & 3.097 & 2.243 \\
\hline \multirow{9}{*}{31} & \multirow{3}{*}{$\mathbf{0}$} & $\overline{0}$ & 5.005 & 2.978 & 2.271 & 4.719 & 2.819 & 2.16 \\
\hline & & $\mathbf{N}_{\mathrm{c}} / \mathbf{2}$ & 5.132 & 3.053 & 2.305 & 4.839 & 2.89 & 2.188 \\
\hline & & $\mathbf{N}_{\mathrm{c}}$ & 5.258 & 3.126 & 2.343 & $\begin{array}{l}4.959 \\
\end{array}$ & 2.959 & 2.221 \\
\hline & \multirow{3}{*}{$\Phi / 2$} & $\overline{0}$ & 10.986 & 4.657 & 3.063 & 10.294 & 4.396 & 2.899 \\
\hline & & $\mathrm{N}_{\mathrm{c}} / 2$ & 11.173 & 4.739 & 3.114 & 10.471 & 4.473 & 2.947 \\
\hline & & $\mathbf{N}_{\mathrm{c}}$ & 11.359 & 4.82 & 3.163 & 10.649 & 4.550 & 2.993 \\
\hline & \multirow{3}{*}{$\Phi$} & 0 & - & 8.767 & 4.437 & -- & 8.233 & 4.192 \\
\hline & & $\mathbf{N}_{\mathrm{c}} / 2$ & -- & 8.876 & 4.485 & -- & 8.336 & 4.237 \\
\hline & & $\mathbf{N}_{\mathrm{c}}$ & - & 8.985 & 4.532 & -- & 8.440 & 4.282 \\
\hline \multirow{9}{*}{40} & \multirow{3}{*}{$\mathbf{0}$} & $\overline{0}$ & 9.846 & 4.582 & 3.164 & 9.280 & 4.342 & 3.032 \\
\hline & & $\mathrm{N}_{\mathrm{c}} / 2$ & 10.026 & 4.680 & 3.173 & 9.451 & 4.434 & 3.034 \\
\hline & & $\mathbf{N}_{\mathbf{c}}$ & 10.206 & 4.776 & 3.188 & 9.622 & 4.525 & 3.042 \\
\hline & \multirow{3}{*}{$\Phi / 2$} & $\overline{0}$ & -- & 10.752 & 5.203 & -- & 10.135 & 4.928 \\
\hline & & $\mathrm{N}_{\mathrm{c}} / 2$ & -- & 10.886 & 5.275 & -- & 10.260 & 4.996 \\
\hline & & $\mathbf{N}_{\mathbf{c}}$ & -- & 11.018 & 5.345 & --- & 10.386 & 5.063 \\
\hline & \multirow{3}{*}{$\Phi$} & $\overline{0}$ & -- & -- & 11.607 & -- & -- & 10.939 \\
\hline & & $\mathbf{N}_{\mathrm{c}} / 2$ & -- & -- & 11.647 & -- & -- & 11.027 \\
\hline & & $\mathbf{N}_{\mathbf{c}}$ & -- & -- & 11.788 & -- & -- & 11.113 \\
\hline
\end{tabular}

Here, a detailed parametric study has been conducted to find the variations of seismic passive earth pressure co-efficient with a wide range of variation of parameters like angle of internal friction $(\Phi)$, angle of wall friction $(\delta)$, wall inclination angle $(\alpha)$, cohesion $(\mathrm{c})$, adhesion $\left(\mathrm{c}_{\mathrm{a}}\right)$, seismic acceleration $\left(\mathrm{k}_{\mathrm{h}}\right.$, $\mathrm{k}_{\mathrm{v}}$ ), and the height of retaining wall $(\mathrm{H})$. The values have been optimized for the $\mathrm{i}^{\text {th }}$ slice considering passive pseudo-static state of equilibrium. Variations of parameters considered are detailed below:

$\Phi=20^{\circ}, 30^{\circ}$ and $40^{\circ} ; \delta=0, \Phi / 2$ and $\Phi ; \alpha=+20^{\circ}, 0^{\circ}$ and $-20^{\circ}$; $\mathrm{k}_{\mathrm{h}}=0,0.1$ and $0.2 ; \mathrm{k}_{\mathrm{v}}=0, \mathrm{k}_{\mathrm{h}} / 2, \mathrm{k}_{\mathrm{h}} ; \mathrm{N}_{\mathrm{c}}=0.1,0.2 ; \mathrm{M}_{\mathrm{c}}=0, \mathrm{~N}_{\mathrm{c}} / 2$, $\mathrm{N}_{\mathrm{c}} ; \mathrm{H}=5 \mathrm{~m}, 7.5 \mathrm{~m}$ and $10 \mathrm{~m}$.

Table-2: Passive earth pressure coefficients $\left(\mathrm{K}_{\mathrm{p}}\right)$ for $\mathrm{N}_{\mathrm{c}}=0.1$, $\mathrm{k}_{\mathrm{h}}=0.1$

\begin{tabular}{|c|c|c|c|c|c|}
\hline \multirow{2}{*}{$\Phi$} & \multirow{2}{*}{$\delta$} & \multirow{2}{*}{$\mathbf{M}_{\mathbf{c}}$} & \multicolumn{3}{|c|}{$\mathbf{k}_{\mathrm{v}}=\mathbf{k}_{\mathrm{h}}$} \\
\hline & & & $\alpha=-20^{\circ}$ & $\boldsymbol{\alpha}=\mathbf{0}^{\circ}$ & $\alpha=+20^{\circ}$ \\
\hline \multirow{9}{*}{20} & \multirow{3}{*}{$\mathbf{0}$} & 0 & 2.489 & 1.789 & 1.527 \\
\hline & & $\mathbf{N}_{\mathrm{c}} / 2$ & 2.571 & 1.838 & 1.551 \\
\hline & & $\mathbf{N}_{\mathrm{c}}$ & 2.651 & 1.886 & 1.588 \\
\hline & \multirow{3}{*}{$\Phi / 2$} & $\overline{0}$ & 3.48 & 2.008 & 1.765 \\
\hline & & $\mathrm{N}_{\mathrm{c}} / 2$ & 3.572 & 2.248 & 1.797 \\
\hline & & $\mathbf{N}_{\mathbf{c}}$ & 3.663 & 2.294 & 1.826 \\
\hline & \multirow{3}{*}{$\Phi$} & 0 & 5.350 & 2.806 & 2.060 \\
\hline & & $\mathbf{N}_{\mathrm{c}} / 2$ & 5.468 & 2.855 & 2.086 \\
\hline & & $\mathbf{N}_{c}$ & 5.583 & 2.905 & 2.110 \\
\hline \multirow{9}{*}{30} & \multirow{3}{*}{$\mathbf{0}$} & 0 & 4.432 & 2.661 & 2.050 \\
\hline & & $\mathbf{N}_{\mathrm{c}} / 2$ & 4.546 & 2.728 & 2.073 \\
\hline & & $\mathbf{N}_{\mathbf{c}}$ & 4.659 & 2.793 & 2.099 \\
\hline & \multirow{3}{*}{$\Phi / 2$} & $\overline{0}$ & 9.594 & 4.135 & 2.7347 \\
\hline & & $\mathbf{N}_{\mathrm{c}} / 2$ & 9.766 & 4.208 & 2.780 \\
\hline & & $\mathbf{N}_{c}$ & 9.380 & 4.280 & 2.824 \\
\hline & \multirow{3}{*}{$\Phi$} & 0 & -- & 7.699 & 3.946 \\
\hline & & $\mathbf{N}_{\mathrm{c}} / 2$ & -- & 7.797 & 3.989 \\
\hline & & $\mathbf{N}_{\mathbf{c}}$ & -- & 7.895 & 4.031 \\
\hline \multirow{9}{*}{40} & \multirow{3}{*}{$\mathbf{0}$} & $\overline{0}$ & 8.713 & 4.101 & 2.869 \\
\hline & & $\mathbf{N}_{\mathrm{c}} / 2$ & 8.876 & 4.188 & 2.895 \\
\hline & & $N_{c}$ & 9.035 & 4.274 & 2.897 \\
\hline & \multirow{3}{*}{$\Phi / 2$} & 0 & -- & 9.814 & 4.653 \\
\hline & & $\mathbf{N}_{\mathrm{c}} / 2$ & -- & 9.634 & 4.718 \\
\hline & & $\mathbf{N}_{\mathrm{c}}$ & -- & 9.753 & 4.781 \\
\hline & \multirow{3}{*}{$\Phi$} & 0 & -- & -- & 10.268 \\
\hline & & $\mathbf{N}_{\mathrm{c}} / 2$ & -- & -- & 10.351 \\
\hline & & $\mathbf{N}_{\mathrm{c}}$ & -- & -- & 10.434 \\
\hline
\end{tabular}


Table-3: Passive earth pressure coefficients $\left(\mathrm{K}_{\mathrm{p}}\right)$ for $\mathrm{N}_{\mathrm{c}}=0.1$, $\mathrm{k}_{\mathrm{h}}=0.2$

\begin{tabular}{|c|c|c|c|c|c|c|c|c|}
\hline \multirow{2}{*}{$\Phi$} & \multirow{2}{*}{$\boldsymbol{\delta}$} & \multirow{2}{*}{$\mathbf{M}_{\mathbf{c}}$} & \multicolumn{3}{|c|}{$k_{v}=0$} & \multicolumn{3}{|c|}{$k_{v}=k_{h} / 2$} \\
\hline & & & $\begin{array}{l}\boldsymbol{\alpha}=- \\
\mathbf{2 0}^{\circ}\end{array}$ & $\boldsymbol{\alpha}=\mathbf{0}^{\circ}$ & $\begin{array}{l}\alpha= \\
+20^{\circ}\end{array}$ & $\begin{array}{l}\alpha=- \\
20^{\circ}\end{array}$ & $\boldsymbol{\alpha}=\mathbf{0}^{\circ}$ & $\alpha=\mathbf{2 0}^{\circ}$ \\
\hline \multirow{9}{*}{20} & \multirow{3}{*}{ 0 } & $\mathbf{0}$ & 2.450 & 1.814 & 1.572 & 2.115 & 1.588 & 1.385 \\
\hline & & $\mathbf{N}_{\mathrm{c}} / 2$ & 2.532 & 1.861 & 1.593 & 2.187 & 1.629 & 1.400 \\
\hline & & $\mathbf{N}_{\mathbf{c}}$ & 2.613 & 1.907 & 1.626 & 2.257 & 1.669 & 1.416 \\
\hline & \multirow{3}{*}{$\Phi / 2$} & 0 & 3.311 & 2.267 & 1.780 & 2.815 & 1.887 & 1.551 \\
\hline & & $\mathbf{N}_{\mathrm{c}} / \mathbf{2}$ & 3.405 & 2.221 & 1.808 & 2.898 & 1.927 & 1.576 \\
\hline & & $\mathbf{N}_{\mathbf{c}}$ & 3.498 & 2.267 & 1.834 & 2.980 & 1.966 & 1.599 \\
\hline & \multirow{3}{*}{$\Phi$} & 0 & 4.896 & 2.704 & 2.035 & 4.077 & 2.320 & 1.768 \\
\hline & & $\mathrm{N}_{\mathrm{c}} / 2$ & 5.016 & 2.752 & 2.058 & 4.182 & 2.362 & 1.787 \\
\hline & & $\mathbf{N}_{\mathbf{c}}$ & 5.135 & 2.801 & 2.080 & 4.287 & 2.405 & 1.805 \\
\hline \multirow{9}{*}{30} & \multirow{3}{*}{ 0 } & $\mathbf{0}$ & 4.516 & 2.775 & 2.155 & 3.938 & 2.455 & 1.936 \\
\hline & & $\mathrm{N}_{\mathrm{c}} / 2$ & 4.636 & 2.845 & 2.181 & 4.046 & 2.516 & 1.951 \\
\hline & & $\mathbf{N}_{\mathbf{c}}$ & 4.756 & 2.913 & 2.221 & 4.152 & 2.577 & 1.969 \\
\hline & \multirow{3}{*}{$\Phi / 2$} & $\mathbf{0}$ & 9.453 & 4.212 & 2.839 & 8.053 & 3.685 & 2.506 \\
\hline & & $\mathrm{N}_{\mathrm{c}} / 2$ & 9.635 & 4.289 & 2.885 & 8.216 & 3.754 & 2.547 \\
\hline & & $\mathbf{N}_{\mathbf{c}}$ & 9.816 & 4.365 & 2.930 & 8.379 & 3.821 & 2.587 \\
\hline & \multirow{3}{*}{$\Phi$} & 0 & -- & 7.641 & 4.016 & -- & 6.564 & 3.521 \\
\hline & & $\mathbf{N}_{\mathrm{c}} / 2$ & -- & 7.746 & 4.060 & -- & 6.657 & 3.560 \\
\hline & & $\mathbf{N}_{\mathbf{c}}$ & -- & 7.847 & 4.104 & -- & 6.750 & 3.598 \\
\hline \multirow{9}{*}{40} & \multirow{3}{*}{ 0 } & $\mathbf{0}$ & 9.062 & 4.346 & 3.047 & 7.923 & 3.863 & 2.783 \\
\hline & & $\mathrm{N}_{\mathrm{c}} / 2$ & 9.238 & 4.440 & 3.054 & 8.081 & 3.946 & 2.778 \\
\hline & & $\mathbf{N}_{\mathbf{c}}$ & 9.414 & 4.532 & 3.091 & 8.238 & 4.028 & 2.803 \\
\hline & \multirow{3}{*}{$\Phi / 2$} & $\mathbf{0}$ & -- & 9.860 & 4.903 & -- & 8.62 & 4.352 \\
\hline & & $\mathrm{N}_{\mathrm{c}} / 2$ & -- & 9.992 & 4.972 & -- & 8.735 & 4.412 \\
\hline & & $\mathbf{N}_{\mathbf{c}}$ & -- & 10.124 & 5.038 & -- & 8.851 & 4.472 \\
\hline & \multirow{3}{*}{$\Phi$} & $\mathbf{0}$ & -- & -- & 10.615 & -- & -- & 9.275 \\
\hline & & $\mathrm{N}_{\mathrm{c}} / 2$ & -- & -- & 10.704 & -- & -- & 9.353 \\
\hline & & $\mathbf{N}_{\mathbf{c}}$ & -- & -- & 10.792 & -- & -- & 9.432 \\
\hline
\end{tabular}

Table-4: Passive earth pressure coefficients $\left(\mathrm{K}_{\mathrm{p}}\right)$ for $\mathrm{N}_{\mathrm{c}}=0.1$, $\mathrm{k}_{\mathrm{h}}=0.2$

\begin{tabular}{|c|c|c|c|c|c|}
\hline \multirow{2}{*}{$\Phi$} & \multirow{2}{*}{$\delta$} & \multirow{2}{*}{$\mathbf{M}_{\mathbf{c}}$} & \multicolumn{3}{|c|}{$\mathbf{k}_{v}=\mathbf{k}_{\mathrm{h}}$} \\
\hline & & & $\alpha=-20^{\circ}$ & $\alpha=0^{\circ}$ & $\alpha=+20^{\circ}$ \\
\hline \multirow{9}{*}{20} & \multirow{3}{*}{ O } & $\overline{0}$ & $\begin{array}{l}1.774 \\
\end{array}$ & 1.426 & 1.200 \\
\hline & & $\mathbf{N}_{\mathrm{c}} / 2$ & 1.835 & 1.489 & 1.206 \\
\hline & & $\mathbf{N}_{\mathbf{c}}$ & 1.895 & 1.550 & 1.215 \\
\hline & \multirow{3}{*}{$\Phi / 2$} & $\overline{0}$ & 2.310 & 1.593 & 1.315 \\
\hline & & $\mathrm{N}_{\mathrm{c}} / 2$ & 2.380 & 1.627 & 1.337 \\
\hline & & $\overline{\mathbf{N}_{\mathbf{c}}}$ & 2.450 & 1.659 & 1.357 \\
\hline & \multirow{3}{*}{$\Phi$} & 0 & 3.243 & 1.929 & 1.495 \\
\hline & & $\mathrm{N}_{\mathrm{c}} / 2$ & 3.334 & 1.965 & 1.510 \\
\hline & & $\mathbf{N}_{\mathrm{c}}$ & 3.424 & 2.000 & 1.524 \\
\hline \multirow{9}{*}{30} & \multirow{3}{*}{ 0 } & 0 & 3.357 & 2.133 & 1.715 \\
\hline & & $\mathrm{N}_{\mathrm{c}} / 2$ & 3.451 & 2.186 & 1.723 \\
\hline & & $\mathbf{N}_{\mathbf{c}}$ & 3.544 & 2.239 & 1.732 \\
\hline & \multirow{3}{*}{$\Phi / 2$} & 0 & 6.623 & 3.156 & 2.172 \\
\hline & & $\mathrm{N}_{\mathrm{c}} / 2$ & 6.772 & 3.215 & 2.208 \\
\hline & & $\mathbf{N}_{\mathrm{c}}$ & 6.920 & 3.274 & 2.242 \\
\hline & \multirow{3}{*}{$\Phi$} & 0 & -- & 5.474 & 3.024 \\
\hline & & $\mathrm{N}_{\mathrm{c}} / 2$ & -- & 5.557 & 3.058 \\
\hline & & $\mathbf{N}_{\mathrm{c}}$ & -- & 5.639 & 3.091 \\
\hline \multirow{9}{*}{40} & \multirow{3}{*}{ o } & $\overline{0}$ & 6.776 & 3.379 & 2.490 \\
\hline & & $\mathrm{N}_{\mathrm{c}} / 2$ & 6.917 & 3.452 & 2.503 \\
\hline & & $\mathbf{N}_{\mathrm{c}}$ & 7.054 & 3.524 & 2.517 \\
\hline & \multirow{3}{*}{$\Phi / 2$} & 0 & -- & 7.369 & 3.798 \\
\hline & & $\mathrm{N}_{\mathrm{c}} / 2$ & -- & 7.473 & 3.851 \\
\hline & & $\mathbf{N}_{\mathrm{c}}$ & -- & 7.576 & 3.903 \\
\hline & \multirow{3}{*}{$\Phi$} & 0 & -- & -- & 7.922 \\
\hline & & $\mathrm{N}_{\mathrm{c}} / 2$ & -- & -- & 7.993 \\
\hline & & $\mathbf{N}_{\mathbf{c}}$ & -- & -- & 8.064 \\
\hline
\end{tabular}


Table-5: Passive earth pressure coefficients $\left(\mathrm{K}_{\mathrm{p}}\right)$ for $\mathrm{N}_{\mathrm{c}}=0.2$, $\mathrm{k}_{\mathrm{h}}=0.1$

\begin{tabular}{|c|c|c|c|c|c|c|c|c|}
\hline \multirow[b]{2}{*}{$\Phi$} & \multirow{2}{*}{$\boldsymbol{\delta}$} & \multirow{2}{*}{$\mathbf{M}_{\mathrm{c}}$} & \multicolumn{3}{|c|}{$\mathbf{k}_{v}=\mathbf{0}$} & \multicolumn{3}{|c|}{$\mathbf{k}_{\mathrm{v}}=\mathrm{k}_{\mathrm{h}} / \mathbf{2}$} \\
\hline & & & $\begin{array}{l}\boldsymbol{\alpha}=- \\
\mathbf{2 0}^{\circ}\end{array}$ & $\boldsymbol{\alpha}=\boldsymbol{0}^{\circ}$ & $\begin{array}{l}\alpha= \\
+20^{\circ}\end{array}$ & $\begin{array}{l}\alpha=-=- \\
20^{\circ}\end{array}$ & $\boldsymbol{\alpha}=\mathbf{0}^{\circ}$ & $\begin{array}{l}\alpha= \\
+{ }^{\alpha} 0^{\circ}\end{array}$ \\
\hline \multirow{9}{*}{20} & \multirow{3}{*}{ o } & 0 & 2.995 & 2.117 & 1.746 & 2.823 & 2.001 & 1.691 \\
\hline & & $\mathrm{N}_{\mathrm{c}} / 2$ & 3.171 & 2.219 & 1.876 & 2.989 & 2.097 & 1.773 \\
\hline & & $N_{c}$ & 3.340 & 2.316 & 1.942 & 3.149 & 2.189 & 1.836 \\
\hline & \multirow{3}{*}{$\Phi / 2$} & 0 & 4.168 & 2.585 & 2.052 & 3.916 & 2.44 & 1.939 \\
\hline & & $\mathrm{Nc} / 2$ & 4.368 & 2.687 & 2.112 & 4.106 & 2.536 & 1.996 \\
\hline & & $\mathbf{N}_{\mathrm{c}}$ & 4.465 & 2.785 & 2.167 & 4.293 & 2.629 & 2.048 \\
\hline & \multirow{3}{*}{$\Phi$} & 0 & 6.404 & 3.288 & 2.375 & 5.993 & 3.097 & 2.243 \\
\hline & & $\mathrm{N}_{\mathrm{c}} / \mathbf{2}$ & 6.659 & 3.396 & 2.428 & 6.235 & 3.198 & 2.292 \\
\hline & & $\mathbf{N}_{\mathrm{c}}$ & 6.911 & 3.502 & 2.478 & 6.474 & 3.298 & 2.339 \\
\hline \multirow{9}{*}{30} & \multirow{3}{*}{0} & 0 & 5.258 & 3.126 & 2.343 & 4.959 & 2.959 & 2.221 \\
\hline & & $\mathrm{N}_{\mathrm{c}} / \mathbf{2}$ & 5.504 & 3.267 & 2.447 & $\begin{array}{l}5.192 \\
\end{array}$ & 3.093 & 2.312 \\
\hline & & $\mathbf{N}_{\mathrm{c}}$ & 5.745 & 3.403 & 2.566 & 5.42 & 3.222 & 2.421 \\
\hline & \multirow{3}{*}{$\Phi / 2$} & 0 & 11.359 & 4.82 & 3.163 & $\begin{array}{l}10.649 \\
\end{array}$ & 4.55 & 2.993 \\
\hline & & $\mathbf{N}_{\mathrm{c}} / \mathbf{2}$ & 11.733 & 4.977 & 3.256 & 11.003 & 4.699 & 3.082 \\
\hline & & $N_{c}$ & 12.104 & 5.132 & 3.346 & 11.35 & 4.846 & 3.167 \\
\hline & \multirow{3}{*}{$\Phi$} & 0 & -- & 8.985 & 4.532 & -- & 8.440 & 4.282 \\
\hline & & $\mathbf{N}_{c} / 2$ & -- & 9.200 & 4.625 & -- & 8.646 & 4.370 \\
\hline & & $\mathbf{N}_{\mathrm{c}}$ & -- & 9.409 & 4.716 & -- & 8.846 & 4.455 \\
\hline \multirow{9}{*}{40} & \multirow{3}{*}{0} & 0 & 10.206 & 4.776 & 3.188 & 9.622 & 4.525 & 3.042 \\
\hline & & $\mathbf{N}_{\mathrm{c}} / \mathbf{2}$ & 10.561 & 4.964 & 2.258 & 9.956 & 4.703 & 3.099 \\
\hline & & $\mathbf{N}_{\mathbf{c}}$ & 10.912 & 5.145 & 3.362 & 10.289 & 4.875 & 3.189 \\
\hline & \multirow{3}{*}{$\Phi / 2$} & 0 & -- & 11.018 & 5.345 & -- & 10.386 & 5.063 \\
\hline & & $\mathbf{N}_{\mathrm{c}} / \mathbf{2}$ & - & 11.282 & 5.483 & -- & 10.636 & 5.193 \\
\hline & & $\mathbf{N}_{\mathrm{c}}$ & - & 11.546 & 5.617 & -- & 10.887 & 5.32 \\
\hline & \multirow{3}{*}{$\Phi$} & 0 & -- & -- & 11.788 & -- & -- & 11.113 \\
\hline & & $N_{c} / 2$ & -- & -- & 11.969 & -- & -- & 11.285 \\
\hline & & $\mathbf{N}_{\mathrm{c}}$ & -- & -- & 12.15 & -- & -- & 11.456 \\
\hline
\end{tabular}

Table-6: Passive earth pressure coefficients $\left(\mathrm{K}_{\mathrm{p}}\right)$ for $\mathrm{N}_{\mathrm{c}}=0.2$, $\mathrm{k}_{\mathrm{h}}=0.1$

\begin{tabular}{|c|c|c|c|c|c|}
\hline \multirow{2}{*}{$\Phi$} & \multirow{2}{*}{$\delta$} & \multirow{2}{*}{$\mathbf{M}_{\mathbf{c}}$} & \multicolumn{3}{|c|}{$k_{v}=k_{h}$} \\
\hline & & & $\alpha=-20^{\circ}$ & $\boldsymbol{\alpha}=\mathbf{0}^{\circ}$ & $\alpha=+20^{\circ}$ \\
\hline \multirow{9}{*}{20} & \multirow{3}{*}{$\mathbf{0}$} & 0 & 2.651 & 1.886 & 1.588 \\
\hline & & $\mathrm{N}_{\mathrm{c}} / 2$ & 2.807 & 1.976 & 1.669 \\
\hline & & $\mathbf{N}_{\mathbf{c}}$ & 2.958 & 2.062 & 1.730 \\
\hline & \multirow{3}{*}{$\Phi / 2$} & 0 & 3.663 & 2.294 & 1.826 \\
\hline & & $\mathrm{Nc} / 2$ & 3.843 & 2.385 & 1.880 \\
\hline & & $\mathbf{N}_{\mathrm{c}}$ & 4.019 & 2.472 & 1.929 \\
\hline & \multirow{3}{*}{$\Phi$} & 0 & 5.583 & 2.905 & 2.11 \\
\hline & & $\mathrm{N}_{\mathrm{c}} / 2$ & 5.81 & 3.000 & 2.157 \\
\hline & & $\mathbf{N}_{\mathrm{c}}$ & 6.034 & 3.094 & 2.200 \\
\hline \multirow{9}{*}{30} & \multirow{3}{*}{$\mathbf{0}$} & 0 & 4.659 & 2.793 & 2.099 \\
\hline & & $\mathrm{N}_{\mathrm{c}} / 2$ & 4.879 & 2.919 & 2.179 \\
\hline & & $\mathbf{N}_{\mathrm{c}}$ & 5.096 & 3.040 & 2.274 \\
\hline & \multirow{3}{*}{$\Phi / 2$} & 0 & 9.38 & 4.280 & 2.824 \\
\hline & & $\mathrm{N}_{\mathrm{c}} / 2$ & 10.26 & 4.421 & 2.908 \\
\hline & & $\mathbf{N}_{\mathrm{c}}$ & 10.595 & 4.559 & 2.968 \\
\hline & \multirow{3}{*}{$\Phi$} & 0 & -- & 7.895 & 4.031 \\
\hline & & $\mathrm{N}_{\mathrm{c}} / 2$ & -- & 8.086 & 4.114 \\
\hline & & $\mathbf{N}_{\mathrm{c}}$ & -- & 8.277 & 4.195 \\
\hline \multirow{9}{*}{40} & \multirow{3}{*}{0} & 0 & 9.035 & 4.274 & 2.897 \\
\hline & & $\mathrm{N}_{\mathrm{c}} / 2$ & 9.351 & 4.442 & 2.963 \\
\hline & & $\mathbf{N}_{\mathrm{c}}$ & 9.667 & 4.605 & 3.018 \\
\hline & \multirow{3}{*}{$\Phi / 2$} & 0 & -- & 9.753 & 4.781 \\
\hline & & $\mathbf{N}_{\mathrm{c}} / 2$ & -- & 9.990 & 4.904 \\
\hline & & $\mathbf{N}_{\mathrm{c}}$ & -- & 10.228 & 5.023 \\
\hline & \multirow{3}{*}{$\Phi$} & 0 & -- & -- & 10.434 \\
\hline & & $\mathbf{N}_{\mathrm{c}} / 2$ & -- & -- & 10.599 \\
\hline & & $\mathbf{N}_{\mathrm{c}}$ & -- & -- & 10.761 \\
\hline
\end{tabular}


Table-7: Passive Earth pressure coefficients $\left(\mathrm{K}_{\mathrm{p}}\right)$ for $\mathrm{N}_{\mathrm{c}}=0.2$, $\mathrm{k}_{\mathrm{h}}=0.2$

\begin{tabular}{|c|c|c|c|c|c|c|c|c|}
\hline \multirow{2}{*}{$\Phi$} & \multirow{2}{*}{$\delta$} & \multirow{2}{*}{$\mathbf{M}_{\mathbf{c}}$} & \multicolumn{3}{|c|}{$\mathbf{k}_{\mathrm{v}}=\mathbf{0}$} & \multicolumn{3}{|c|}{$k_{v}=k_{h} / 2$} \\
\hline & & & $\alpha=-20^{\circ}$ & $\boldsymbol{\alpha}=\mathbf{0}^{\circ}$ & $\begin{array}{l}\alpha= \\
+20^{\circ} \\
\end{array}$ & $\begin{array}{l}\alpha=- \\
20^{\circ}\end{array}$ & $\boldsymbol{\alpha}=\mathbf{0}$ & $\begin{array}{l}= \\
+20^{\circ} \\
\end{array}$ \\
\hline \multirow{9}{*}{20} & \multirow{3}{*}{ 0 } & 0 & 2.613 & 1.907 & 1.626 & 2.257 & 1.669 & 1.416 \\
\hline & & $\mathrm{N}_{\mathrm{d}} / 2$ & 2.770 & 1.995 & 1.701 & 2.395 & 1.745 & 1.468 \\
\hline & & $\mathbf{N}_{\mathbf{c}}$ & 2.923 & 2.080 & 1.757 & 2.529 & 1.817 & 1.530 \\
\hline & \multirow{3}{*}{$\Phi / 2$} & $\overline{0}$ & 3.498 & 2.267 & 1.834 & 2.980 & 1.966 & 1.599 \\
\hline & & $\mathrm{N}_{\mathrm{c}} / 2$ & 3.681 & 2.355 & 1.883 & 3.140 & 2.042 & 1.640 \\
\hline & & $\mathbf{N}_{\mathbf{c}}$ & 3.860 & 2.441 & 1.927 & 3.297 & 2.116 & 1.678 \\
\hline & \multirow{3}{*}{$\Phi$} & $\overline{0}$ & 5.135 & 2.801 & 2.080 & 4.287 & 2.405 & 1.805 \\
\hline & & $\mathrm{N}_{\mathrm{c}} / 2$ & 5.367 & 2.896 & 2.121 & 4.496 & 2.487 & 1.839 \\
\hline & & $\mathbf{N}_{\mathbf{c}}$ & 5.599 & 2.989 & 2.160 & 4.696 & 2.567 & 1.872 \\
\hline \multirow{9}{*}{30} & \multirow{3}{*}{ 0 } & 0 & 4.756 & 2.913 & 2.099 & 4.152 & 2.577 & 1.969 \\
\hline & & $\mathrm{N}_{\mathrm{c}} / 2$ & 4.991 & 3.045 & 2.179 & 4.361 & 2.693 & 2.032 \\
\hline & & $\mathbf{N}_{\mathrm{c}}$ & 5.220 & 3.172 & 2.274 & 4.565 & 2.806 & 2.109 \\
\hline & \multirow{3}{*}{$\Phi / 2$} & 0 & 9.816 & 4.365 & 2.930 & 8.379 & 3.821 & 2.587 \\
\hline & & $\mathrm{N}_{\mathrm{c}} / 2$ & 10.3178 & 4.513 & 2.016 & 8.702 & 3.952 & 2.663 \\
\hline & & $\mathbf{N}_{\mathrm{c}}$ & 10.533 & 4.660 & 3.097 & 9.019 & 4.082 & 2.735 \\
\hline & \multirow{3}{*}{$\Phi$} & $\overline{0}$ & -- & 7.847 & 4.104 & -- & 6.750 & 3.598 \\
\hline & & $\mathrm{N}_{\mathrm{c}} / 2$ & -- & 8.050 & 4.190 & -- & 6.934 & 3.674 \\
\hline & & $\mathbf{N}_{\mathbf{c}}$ & -- & 8.252 & 4.273 & -- & 7.111 & 3.747 \\
\hline \multirow{9}{*}{40} & \multirow{3}{*}{$\mathbf{0}$} & $\overline{0}$ & 9.414 & 4.532 & 3.091 & 8.238 & 4.028 & 2.803 \\
\hline & & $\mathrm{N}_{\mathrm{c}} / 2$ & 9.759 & 4.712 & 3.150 & 8.547 & 4.189 & 2.836 \\
\hline & & $\mathbf{N}_{\mathbf{c}}$ & 10.097 & 4.887 & 3.240 & 8.853 & 4.345 & 2.901 \\
\hline & \multirow{3}{*}{$\Phi / 2$} & 0 & -- & 10.124 & 5.038 & -- & 8.851 & 4.472 \\
\hline & & $\mathrm{N}_{\mathrm{c}} / 2$ & -- & 10.382 & 5.170 & -- & 9.082 & 4.588 \\
\hline & & $\mathbf{N}_{\mathrm{c}}$ & -- & 10.639 & 5.298 & -- & 9.313 & 4.702 \\
\hline & \multirow{3}{*}{$\Phi$} & 0 & -- & -- & 10.792 & -- & -- & 9.432 \\
\hline & & $\mathrm{N}_{\mathrm{c}} / 2$ & -- & -- & 10.967 & -- & -- & 9.589 \\
\hline & & $\mathbf{N}_{\mathrm{c}}$ & -- & -- & 11.142 & -- & -- & 9.747 \\
\hline
\end{tabular}

Table-8: Passive Earth pressure coefficients $\left(\mathrm{K}_{\mathrm{p}}\right)$ for $\mathrm{N}_{\mathrm{c}}=0.2$, $\mathrm{k}_{\mathrm{h}}=0.2$

\begin{tabular}{|c|c|c|c|c|c|}
\hline & \multirow{2}{*}{$\delta$} & \multirow{2}{*}{$\mathbf{M}_{\mathbf{c}}$} & \multicolumn{3}{|c|}{$\mathbf{k}_{\mathrm{v}}=\mathrm{k}_{\mathrm{h}}$} \\
\hline & & & $\alpha=-20^{\circ}$ & $\alpha=0^{\circ}$ & $\alpha=+20^{\circ}$ \\
\hline \multirow{9}{*}{20} & \multirow{3}{*}{0} & $\overline{0}$ & 1.895 & 1.426 & 1.215 \\
\hline & & $\mathbf{N}_{\mathrm{c}} / \mathbf{2}$ & 2.012 & 1.489 & 1.236 \\
\hline & & $\mathbf{N}_{\mathrm{c}}$ & 2.127 & 1.550 & 1.270 \\
\hline & \multirow{3}{*}{$\Phi / 2$} & 0 & 2.45 & 1.659 & 1.357 \\
\hline & & $\mathbf{N}_{\mathrm{c}} / \mathbf{2}$ & 2.588 & 1.723 & 1.392 \\
\hline & & $\mathbf{N}_{\mathrm{c}}$ & 2.722 & 1.786 & 1.423 \\
\hline & \multirow{3}{*}{$\Phi$} & $\overline{0}$ & 3.424 & 2.000 & 1.524 \\
\hline & & $\mathbf{N}_{\mathrm{c}} / \mathbf{2}$ & 3.605 & 2.069 & 1.552 \\
\hline & & $\mathbf{N}_{\mathrm{c}}$ & 3.780 & 2.137 & 1.578 \\
\hline \multirow{9}{*}{30} & \multirow{3}{*}{ 0 } & $\overline{0}$ & 3.544 & 2.239 & 1.732 \\
\hline & & $\mathbf{N}_{\mathrm{c}} / \mathbf{2}$ & 3.727 & 2.34 & 1.772 \\
\hline & & $\mathbf{N}_{\mathrm{c}}$ & 3.906 & 2.438 & 1.824 \\
\hline & \multirow{3}{*}{$\Phi / 2$} & 0 & 6.920 & 3.274 & 2.242 \\
\hline & & $\mathrm{N}_{\mathrm{c}} / \mathbf{2}$ & 7.208 & 3.388 & 2.308 \\
\hline & & $\mathbf{N}_{\mathrm{c}}$ & 7.489 & 3.501 & 2.371 \\
\hline & \multirow{3}{*}{$\Phi$} & 0 & -- & 5.639 & 3.091 \\
\hline & & $\mathbf{N}_{\mathrm{c}} / \mathbf{2}$ & -- & 5.804 & 3.155 \\
\hline & & $\mathbf{N}_{\mathrm{c}}$ & -- & 5.961 & 3.219 \\
\hline \multirow{9}{*}{40} & \multirow{3}{*}{ 0 } & $\overline{0}$ & 7.054 & 3.524 & 2.517 \\
\hline & & $\mathbf{N}_{\mathrm{c}} / \mathbf{2}$ & 7.328 & 3.665 & 2.528 \\
\hline & & $\mathbf{N}_{\mathrm{c}}$ & 7.600 & 3.802 & 2.569 \\
\hline & \multirow{3}{*}{$\Phi / 2$} & $\overline{0}$ & -- & 7.576 & 3.903 \\
\hline & & $\mathrm{N}_{\mathrm{c}} / \mathbf{2}$ & -- & 7.781 & 4.006 \\
\hline & & $\mathbf{N}_{\mathrm{c}}$ & -- & 7.980 & 4.105 \\
\hline & \multirow{3}{*}{$\Phi$} & 0 & -- & -- & 8.064 \\
\hline & & $\mathbf{N}_{\mathrm{c}} / \mathbf{2}$ & -- & -- & 8.207 \\
\hline & & $\mathbf{N}_{\mathbf{c}}$ & -- & -- & 8.343 \\
\hline
\end{tabular}

\subsection{Effect of Wall Inclination Angle ( $\alpha$ )}

Fig.3 shows the variation of passive earth pressure coefficient with respect to soil friction angle $(\Phi)$ at different wall inclination angles $\left(\alpha=-20^{\circ}, 0^{\circ}, 20^{\circ}\right)$ for $\mathrm{N}_{\mathrm{c}}=0.1, \mathrm{M}_{\mathrm{c}}=\mathrm{N}_{\mathrm{c}}, \delta=$ $\Phi / 2, k_{h}=0.2$ and $k_{v}=k_{h} / 2$. From the plot, it is seen that the magnitude of seismic passive earth pressure co-efficient $\left(\mathrm{K}_{\mathrm{p}}\right)$ decreases with the increase in wall inclination angle $(\alpha)$. 


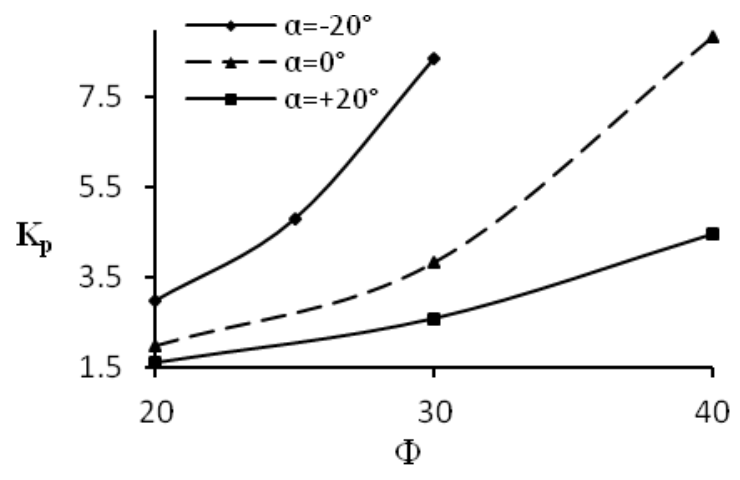

Fig.3 showing the variation of Passive earth pressure coefficient with respect to soil friction angle $(\Phi)$ at different Wall inclination angles $(\alpha=-20,0,20)$ for $\mathrm{N}_{\mathrm{r}}=0.1, \mathrm{M}_{\mathrm{r}}=\mathrm{N}_{\mathrm{r}}, \delta=\Phi / 2, \mathrm{k}_{\mathrm{h}}=0.2$ and $\mathrm{k}_{\mathrm{v}}=\mathrm{k}_{\mathrm{h}} / 2$

The increase in inclination is imposing greater amount of backfill soil, thus the passive resistance is getting reduced. For example, at $\Phi=20^{\circ}, \delta=\Phi / 2$ and $\mathrm{k}_{\mathrm{h}}=0.2, \mathrm{k}_{\mathrm{v}}=\mathrm{k}_{\mathrm{h}} / 2, \mathrm{~N}_{\mathrm{c}}=0.1$, $\mathrm{M}_{\mathrm{c}}=\mathrm{N}_{\mathrm{c}}$, the magnitude of $\mathrm{K}_{\mathrm{p}}$ is 1.966 at $\alpha=0^{\circ}$ which reduces to $\mathrm{K}_{\mathrm{p}}=1.599$ at $\alpha=+20^{\circ}$. Again, at $\Phi=30^{\circ}, \delta=\Phi / 2$ and $\mathrm{k}_{\mathrm{h}}=$ $0.2, \mathrm{k}_{\mathrm{v}}=\mathrm{k}_{\mathrm{h}} / 2, \mathrm{~N}_{\mathrm{c}}=0.1, \mathrm{M}_{\mathrm{c}}=\mathrm{N}_{\mathrm{c}}$, the magnitude of $\mathrm{K}_{\mathrm{p}}$ is 8.379 at $\alpha=-20^{\circ}$ which decreases upto $K_{p}=3.821$ at $\alpha=0^{\circ}$.

\subsection{Effect of Wall Friction Angle ( $\delta$ )}

Fig.4 shows the variations of passive earth pressure coefficient with respect to soil friction angle $(\Phi)$ at different Wall friction angles $(\delta=0, \Phi / 2, \Phi)$ for $\mathrm{N}_{\mathrm{c}}=0.1, \mathrm{M}_{\mathrm{c}}=\mathrm{N}_{\mathrm{c}}, \alpha=20^{\circ}, \mathrm{k}_{\mathrm{h}}=0.2, \mathrm{k}_{\mathrm{v}}=$ $\mathrm{k}_{\mathrm{h}} / 2$.

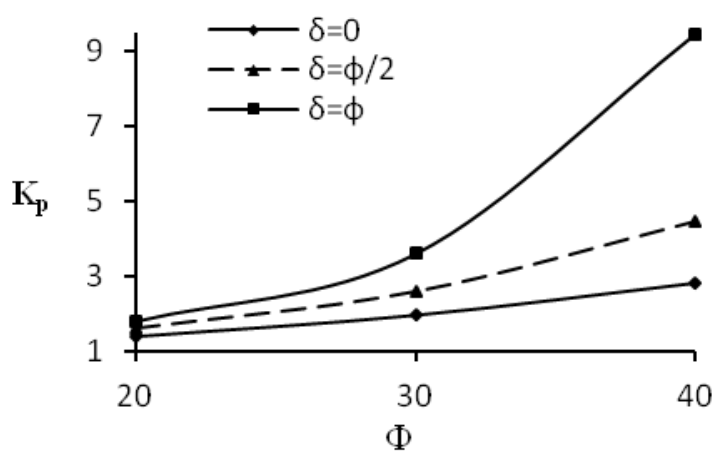

Fig.4 showing the variations of passive earth pressure coefficient with respect to soil friction angle $(\Phi)$ at different Wall friction angles $(\delta=0, \Phi / 2, \Phi)$ for $\mathrm{N}_{\mathrm{r}}=0.1, \mathrm{M}_{\mathrm{r}}=\mathrm{N}_{\mathrm{r},}, \alpha=20^{0}, \mathrm{k}_{\mathrm{h}}=0.2, \mathrm{k}_{\mathrm{v}}=\mathrm{k}_{\mathrm{h}} / 2$

From the plot, it is seen that due to the increase in $\delta$, passive pressure co-efficient $\left(\mathrm{K}_{\mathrm{p}}\right)$ is increased. The friction between wall and soil is increasing the passive resistance. For example, at $\Phi=30^{\circ}, \alpha=+20^{\circ}, \mathrm{N}_{\mathrm{c}=0} 0.1, \mathrm{M}_{\mathrm{c}}=\mathrm{N}_{\mathrm{c},} \mathrm{k}_{\mathrm{h}}=0.2$ and $\mathrm{k}_{\mathrm{v}}=\mathrm{k}_{\mathrm{h}} / 2$, the magnitude of $\mathrm{K}_{\mathrm{p}}$ increases from 2.221 to 2.930 for $\delta=\Phi / 2$ over $\delta=0^{\circ}$. Again, the value is increased upto $\mathrm{K}_{\mathrm{p}}=4.104$ for $\delta$ $=\Phi$ with all other conditions remaining unchanged.

\subsection{Effect of Soil Friction Angle (Ф)}

Fig.5 shows the variations of active earth pressure coefficient $\left(\mathrm{K}_{\mathrm{p}}\right)$ with respect to wall inclination angle $(\alpha)$ for different soil friction angles at $\mathrm{N}_{\mathrm{c}}=0.1, \mathrm{M}_{\mathrm{c}}=\mathrm{N}_{\mathrm{c}}, \mathrm{k}_{\mathrm{h}}=0.2, \mathrm{k}_{\mathrm{v}}=\mathrm{k}_{\mathrm{h}} / 2$.

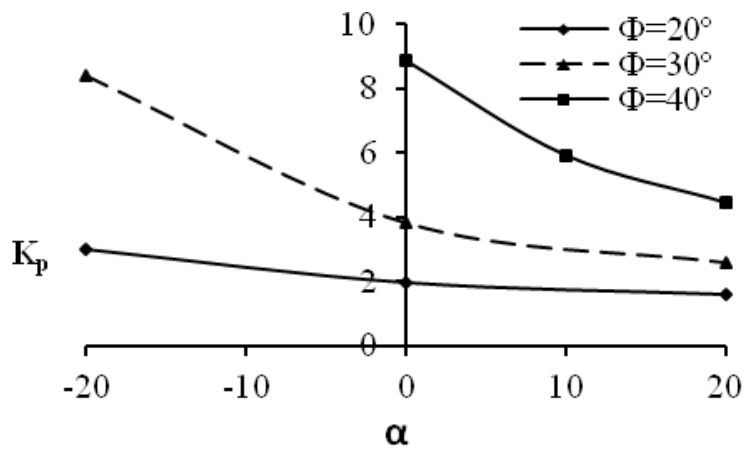

Fig.5 showing the variations of active earth pressure coefficient $\left(\mathrm{K}_{\mathrm{p}}\right)$ with respect to wall inclination angle $(\alpha)$ for different soil friction angles at $\delta=\Phi / 2, \mathrm{~N}_{\mathrm{c}}=0.1$, $\mathrm{M}_{\mathrm{c}}=\mathrm{N}_{\mathrm{c}} \mathrm{k}_{\mathrm{h}}=0.2, \mathrm{k}_{\mathrm{v}}=\mathrm{k}_{\mathrm{h}} / 2$

It is observed that the increase in the value of $\Phi$ increases the passive resistance. The reason behind is that the self resistance of soil increases for higher values of $\Phi$.

\subsection{Effect of kh and kv}

Fig.6 shows the variations of passive earth pressure coefficient $\left(\mathrm{K}_{\mathrm{p}}\right)$ with respect to vertical seismic acceleration coefficient $\left(\mathrm{k}_{\mathrm{v}}\right)$ for $\mathrm{k}_{\mathrm{h}}=0.1,0.2,0.3$ at $\Phi=30^{\circ}, \delta=\Phi / 2, \alpha=+20^{\circ}, \mathrm{N}_{\mathrm{c}}=0.1$, $\mathrm{M}_{\mathrm{c}}=\mathrm{N}_{\mathrm{c}}$ and $\mathrm{k}_{\mathrm{v}}=\mathrm{k}_{\mathrm{h}} / 2$.

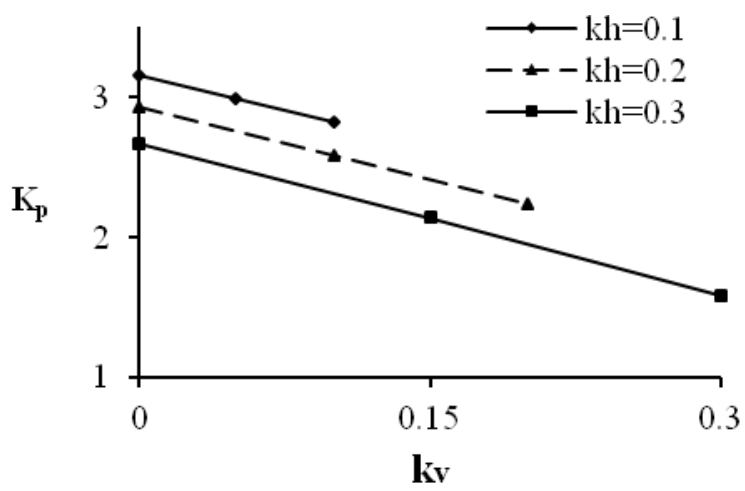

Fig.6 showing the variations of passive earth pressure coefficient $\left(\mathrm{K}_{\mathrm{p}}\right)$ with respect to vertical seismic acceleration coefficient $\left(\mathrm{k}_{\mathrm{v}}\right)$ for $\mathrm{k}_{\mathrm{h}}=0.1,0.2,0.3$ at $\alpha=$ $+20^{\circ}, \Phi=30^{\circ}, \delta=\Phi / 2, \mathrm{~N}_{\mathrm{r}}=0.1, \mathrm{M}_{\mathrm{r}}=\mathrm{N}_{\mathrm{r}}$ and $\mathrm{k}_{\mathrm{v}}=\mathrm{k}_{\mathrm{h}} / 2$

From the figure, it is seen that the magnitude of seismic passive earth pressure co-efficient $\left(\mathrm{K}_{\mathrm{p}}\right)$ is decreased due to the increase in horizontal seismic acceleration $\left(\mathrm{k}_{\mathrm{h}}\right)$. Fig.7 shows the variation of passive earth pressure coefficient $\left(\mathrm{K}_{\mathrm{p}}\right)$ with respect to soil friction angle $(\Phi)$ for different values of $k_{h}$ at 
$\mathrm{k}_{\mathrm{v}}=\mathrm{k}_{\mathrm{h}} / 2, \quad \alpha=+20^{\circ}, \delta=\Phi / 2, \mathrm{~N}_{\mathrm{c}}=0.1$ and $\mathrm{M}_{\mathrm{c}}=\mathrm{N}_{\mathrm{c}}$. With the increase in the value of $\Phi$, the passive resistance increases. For example, at $\Phi=30^{\circ}, \delta=\Phi / 2, \alpha=+20^{\circ}, \mathrm{N}_{\mathrm{c}}=0.2, \mathrm{M}_{\mathrm{c}}=\mathrm{N}_{\mathrm{c}}$ and $\mathrm{k}_{\mathrm{v}}=\mathrm{k}_{\mathrm{h}} / 2$ the magnitude of $\mathrm{K}_{\mathrm{p}}$ is decreased from 3.167 to 2.735 for $\mathrm{k}_{\mathrm{h}}=0.2$ over $\mathrm{k}_{\mathrm{h}}=0.1$.

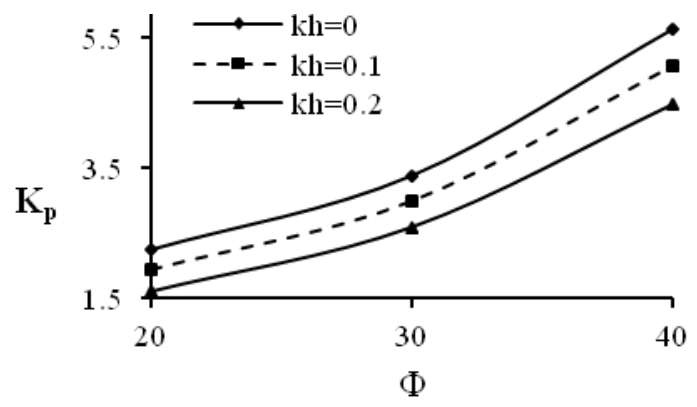

Fig. 7 showing the variation of passive earth pressure coefficient $\left(\mathrm{K}_{\mathrm{p}}\right)$ with respect to soil friction angle $(\Phi)$ for differ ent values of $\mathrm{k}_{\mathrm{h}}$ at $\mathrm{k}_{\mathrm{v}}=\mathrm{k}_{\mathrm{h}} / 2, \alpha=20^{0}, \delta=\Phi / 2$ $\mathrm{N}_{\mathrm{r}}=0.1$ and $\mathrm{M}_{\mathrm{r}}=\mathrm{N}_{\mathrm{r}}$

Again for, $\Phi=20^{\circ}$, the values of $\mathrm{K}_{\mathrm{p}}$ are 1.872 and 2.048 for $\mathrm{k}_{\mathrm{h}}$ $=0.2$ and $\mathrm{k}_{\mathrm{h}}=0.1$ respectively. Fig. 8 Shows the variation of passive earth pressure coefficient $\left(\mathrm{K}_{\mathrm{p}}\right)$ with respect to soil friction angle $(\Phi)$ for different values of $\mathrm{k}_{\mathrm{v}}$ at $\mathrm{k}_{\mathrm{h}}=0.2, \alpha=20^{\circ}$, $\delta=\Phi / 2 \mathrm{~N}_{\mathrm{c}}=0.1$ and $\mathrm{M}_{\mathrm{c}}=\mathrm{N}_{\mathrm{c}}$.

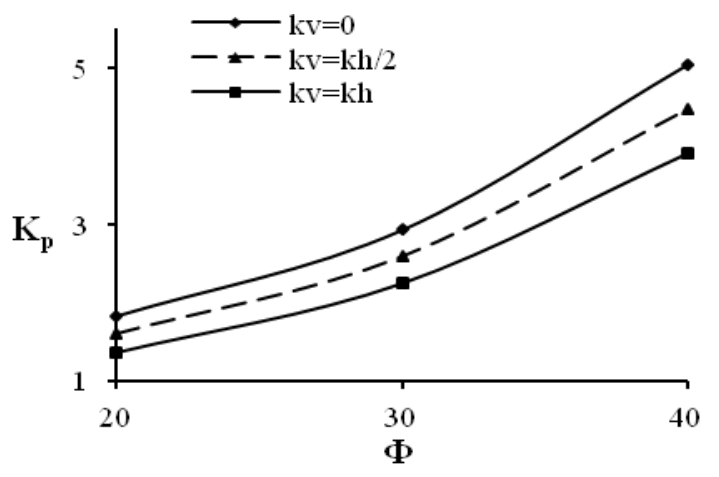

Fig.8 showing the variation of passive earth pressure coefficient $\left(\mathrm{K}_{\mathrm{p}}\right)$ with respect to soil friction angle $(\Phi)$ for different values of $\mathrm{k}_{\mathrm{v}}$ at $\mathrm{k}_{\mathrm{h}}=0.2, \alpha=20^{\circ}, \delta=\Phi / 2$ $\mathrm{N}_{\mathrm{r}}=0.1$ and $\mathrm{M}_{\mathrm{r}}=\mathrm{N}_{\mathrm{r}}$.

From the plot, it is seen that due to the increase in $\mathrm{K}_{\mathrm{v}}$, the passive pressure co-efficient $\left(\mathrm{K}_{\mathrm{p}}\right)$ is going to be decreased. For example, at $\Phi=40^{\circ}, \delta=\Phi / 2, \alpha=+20^{\circ}, \mathrm{N}_{\mathrm{c}}=0.2, \mathrm{M}_{\mathrm{c}}=\mathrm{N}_{\mathrm{c}}$ and $\mathrm{k}_{\mathrm{h}}$ $=0.2$, the magnitude of $\mathrm{K}_{\mathrm{p}}$ is 4.702 at $\mathrm{k}_{\mathrm{v}}=\mathrm{k}_{\mathrm{h}} / 2$ which is reduced upto $\mathrm{K}_{\mathrm{p}}=4.105$ for $\mathrm{k}_{\mathrm{v}}=\mathrm{k}_{\mathrm{h}}$. As the seismic acceleration increases, the disturbance in backfill soil and wall also increases, thus the passive resistance of the backfill soil reduces.

\subsection{Effect of Cohesion (c) and Adhesion (ca)}

Fig.9 shows the variation of passive earth pressure coefficient $\left(\mathrm{K}_{\mathrm{p}}\right)$ with respect to soil friction angle $(\Phi)$ at different values of $\mathrm{N}_{\mathrm{c}}$ for $\mathrm{M}_{\mathrm{c}}=\mathrm{N}_{\mathrm{c}}, \delta=\Phi / 2, \mathrm{k}_{\mathrm{h}}=0.2$ and $\mathrm{k}_{\mathrm{v}}=\mathrm{k}_{\mathrm{h}} / 2$. For the values of $\mathrm{M}_{\mathrm{c}}$ being $0, \mathrm{~N}_{\mathrm{c}} / 2$ and $\mathrm{N}_{\mathrm{c}}$ at $\mathrm{N}_{\mathrm{c}}=0.1, \delta=\Phi / 2, \mathrm{k}_{\mathrm{h}}=0.2, \mathrm{k}_{\mathrm{v}}=\mathrm{k}_{\mathrm{h}} / 2$ and $\alpha=+20^{\circ}$ the value of $\mathrm{K}_{\mathrm{p}}$ gradually increases with the increase of $\mathrm{N}_{\mathrm{c}}$ value.

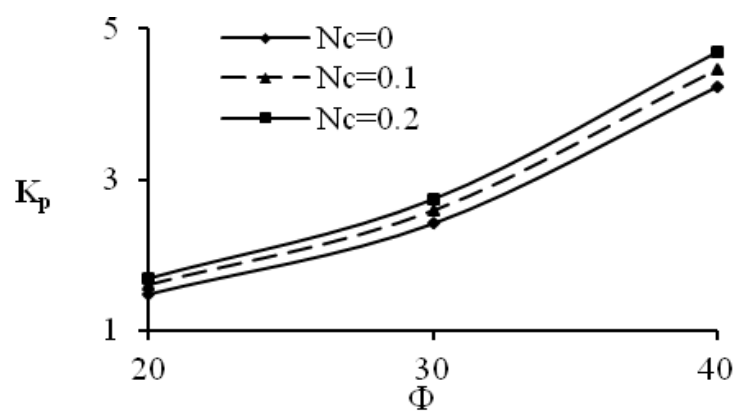

Fig.9 showing the variation of Passive earth pressure coefficient $\left(\mathrm{K}_{\mathrm{p}}\right)$ with respect to soil friction angle $(\Phi)$ at differ ent values of $\mathrm{N}_{\mathrm{c}}$ for $\mathrm{M}_{\mathrm{c}}=\mathrm{N}_{\mathrm{c}}, \delta=\Phi / 2, \alpha=20^{\circ}$, $\mathrm{k}_{\mathrm{h}}=0.2$ andk $_{\mathrm{v}}=\mathrm{k}_{\mathrm{h}} / 2$

Cohesion increases the intermolecular attraction, thus the passive resistance also increases. For example, at $\Phi=30^{\circ}, \mathrm{M}_{\mathrm{c}}$ $=\mathrm{N}_{\mathrm{c}} / 2, \delta=\Phi / 2, \mathrm{k}_{\mathrm{h}}=0.2, \mathrm{k}_{\mathrm{v}}=\mathrm{k}_{\mathrm{h}} / 2$ and $\alpha=+20^{\circ}$ the values of $\mathrm{K}_{\mathrm{p}}$ are 2.547, and 2.663 respectively for $\mathrm{N}_{\mathrm{c}}=0.1$ and 0.2 . Fig. 10 shows the variation of passive earth pressure coefficient $\left(\mathrm{K}_{\mathrm{p}}\right)$ with respect to soil friction angle $(\Phi)$ at different values of $\mathrm{M}_{\mathrm{c}}$ for $\mathrm{N}_{\mathrm{c}}=0.1, \delta=\Phi / 2, \mathrm{k}_{\mathrm{h}}=0.2$ and $\mathrm{k}_{\mathrm{v}}=\mathrm{k}_{\mathrm{h}} / 2$.

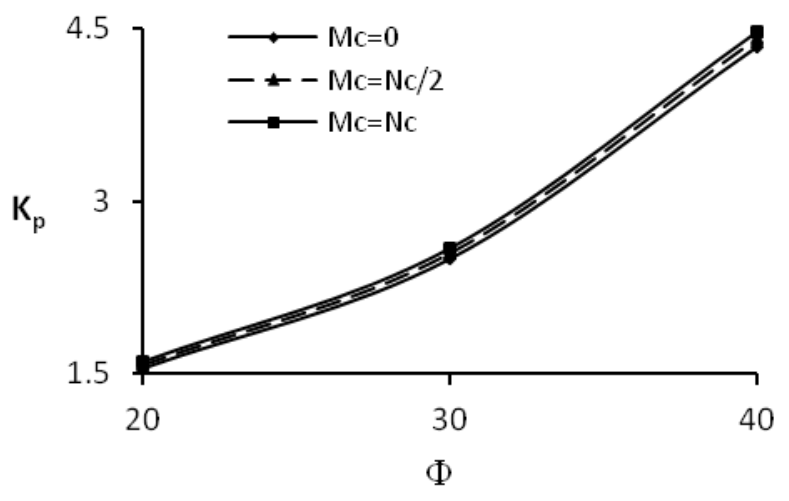

Fig.10 showing the variation of Passive earth pressure coefficient $\left(\mathrm{K}_{\mathrm{p}}\right)$ with respect to soil friction angle $(\Phi)$ at differ ent values of $\mathrm{M}_{\mathrm{c}}$ for $\mathrm{N}_{\mathrm{c}}=0.1, \delta=\Phi / 2, \alpha=20^{\circ}, \mathrm{k}_{\mathrm{h}}=0.2$ and $\mathrm{k}_{\mathrm{v}}=\mathrm{k}_{\mathrm{h}} / 2$

For example, at $\Phi=20^{\circ}, \mathrm{N}_{\mathrm{c}}=0.2, \delta=\Phi / 2, \mathrm{k}_{\mathrm{h}}=0.2, \mathrm{k}_{\mathrm{v}}=\mathrm{k}_{\mathrm{h}} / 2$ and $\alpha=+20^{\circ}$ the values of $K_{p}$ is 2.032 for $M_{c}=N_{c} / 2$, whereas the value increases upto 2.109 for $\mathrm{M}_{\mathrm{c}}=\mathrm{N}_{\mathrm{c}}$ all other conditions remaining unchanged. Thus, it is observed that the increased cohesive and adhesive property of soil material enhances the seismic passive resistance of the retaining wall. 


\subsection{Effect of Height (H)}

Fig.11 shows the variation of passive earth pressure coefficient $\left(\mathrm{K}_{\mathrm{p}}\right)$ with respect to soil friction angle $\left(\mathrm{k}_{\mathrm{h}}\right)$ for different heights at $\mathrm{N}_{\mathrm{c}}=0.1, \mathrm{M}_{\mathrm{c}}=\mathrm{N}_{\mathrm{c}}, \delta=\Phi / 2, \mathrm{k}_{\mathrm{h}}=0.2$ and $\mathrm{k}_{\mathrm{v}}=$ $\mathrm{k}_{\mathrm{h}} / 2$. The comparative results have been put considering the height of wall to be $5 \mathrm{~m}, 7.5 \mathrm{~m}$ and $10 \mathrm{~m}$. From the figure it is observed that the value of $\mathrm{K}_{\mathrm{p}}$ gradually decreases with increase in height of retaining wall.

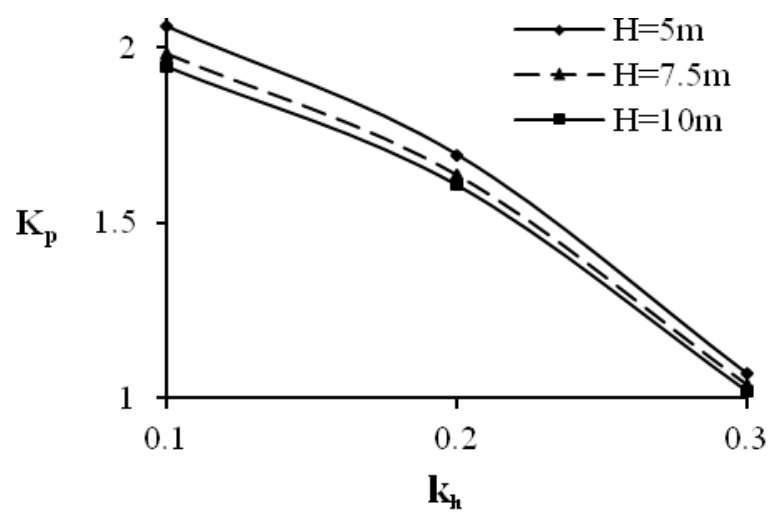

Fig.11 shows the variation of Passive earth pressure coefficient $\left(\mathrm{K}_{\mathrm{p}}\right)$ with respect to soil friction angle $\left(\mathrm{k}_{\mathrm{h}}\right)$ for different heights at $\mathrm{N}_{\mathrm{c}}=0.1, \mathrm{M}_{\mathrm{c}}=\mathrm{N}_{\mathrm{c},} \delta=\Phi / 2, \alpha=20^{\circ}$, $\mathrm{k}_{\mathrm{h}}=0.2$ and $\mathrm{k}_{\mathrm{v}}=\mathrm{k}_{\mathrm{h}} / 2$

It is also seen that the magnitude of $K_{p}$ is increased with the increase in soil friction angle $(\Phi)$ for a constant height of retaining wall. Higher retaining walls support greater amount of backfill and thus the passive resistance is supposed to decrease.

\subsection{Nonlinearity of Failure Surface}

Fig.12 shows the comparison between failure surface of backfill for wall inclination, $\alpha=+20^{\circ}$ at $\Phi=30^{\circ}, \delta=\Phi / 2$, $\mathrm{k}_{\mathrm{h}}=0.2$ and $\mathrm{k}_{\mathrm{v}}=\mathrm{k}_{\mathrm{h}} / 2, \mathrm{~N}_{\mathrm{c}}=0.1$ and $\mathrm{M}_{\mathrm{c}}=\mathrm{N}_{\mathrm{c}}$. It is observed that the failure surface is curvilinear in nature for present analysis.

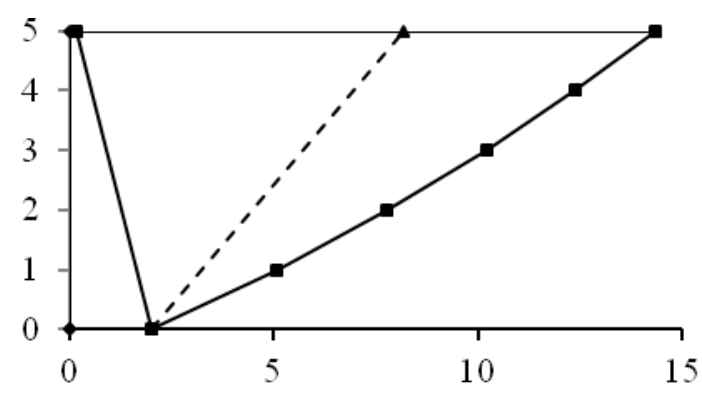

Fig.12 showing the comparison between failure surface of backfill (passive Pseudo-static case) for wall inclination, $\alpha=+20^{\circ}$ at $\Phi=30^{\circ}, \delta=\Phi / 2$, $\mathrm{k}_{\mathrm{h}}=0.2$ and $_{\mathrm{v}}=\mathrm{k}_{\mathrm{h}} / 2, \mathrm{~N}_{\mathrm{r}}=0.1$ and $\mathrm{M}_{\mathrm{r}}=\mathrm{N}_{\mathrm{r}}$.
The failure line is linear for Ghosh and Sengupta (2012) analysis. For example, at $\alpha=+20^{\circ}$ for $\Phi=30^{\circ}, \delta=\Phi / 2, N_{c}=0.1$, $\mathrm{M}_{\mathrm{c}}=\mathrm{N}_{\mathrm{c}}$ and $\mathrm{k}_{\mathrm{h}}=0.2, \mathrm{k}_{\mathrm{v}}=\mathrm{k}_{\mathrm{h}} / 2$ the value of failure surface inclination with the vertical at bottom $63^{\circ}$ and angle at the top is $70^{\circ}$, whereas; at $\alpha=-20^{\circ}$ the value of the inclination of failure surface with vertical at bottom $74^{\circ}$ and angle at the top is $86^{\circ}$. It is seen that the inclination of the failure surface with vertical reduces with the increase in the wall inclination angles when the inclination of the wall is away from the backfill.

\section{COMPARISON OF RESULTS}

Fig.13 shows the comparison of results for variations of passive earth pressure coefficient with respect to soil friction angle $(\Phi)$ at $\alpha=+20^{\circ}, \delta=\Phi / 2, \mathrm{k}_{\mathrm{h}}=0.2$ and $\mathrm{k}_{\mathrm{v}}=\mathrm{k}_{\mathrm{h}} / 2, \mathrm{~N}_{\mathrm{c}}=0.1$ and $\mathrm{M}_{\mathrm{c}}=\mathrm{N}_{\mathrm{c}}$.

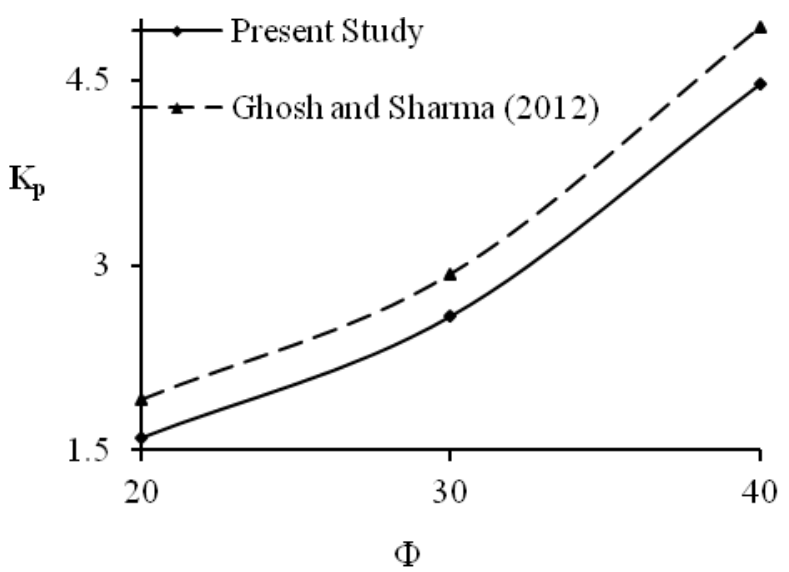

Fig.13 showing the comparison of results for variations of passive earth pressure coefficient with respect to soil friction angle $(\Phi)$ at $\alpha=+20^{\circ}, \delta=\Phi / 2, \mathrm{k}_{\mathrm{h}}=0.2$ and $\mathrm{k}_{\mathrm{v}}=\mathrm{k}_{\mathrm{h}} / 2$, $\mathrm{N}_{\mathrm{r}}=0.1$ and $\mathrm{M}_{\mathrm{r}}=\mathrm{N}_{\mathrm{r}}$.

The present values are comparable with existing earth pressure theories. Here, the graph is plotted to compare the results obtained from present study with the results of Ghosh and Sharma (2012) analysis. The comparison of the values shows that the present value of $\mathrm{K}_{\mathrm{p}}$ is around $5-10 \%$ smaller than the values of Ghosh and Sharma (2012) analysis.

\section{CONCLUSIONS}

In this study, the Horizontal Slices Method of analysis with pseudo-static approach has been considered to determine the seismic passive resistance of the retaining wall with non-linear failure surface. The present study shows that the non-linearity of the failure surface is affecting the evaluation of seismic passive earth pressure. It is also noted that the nature of the failure surface changes with the change in wall inclination angle and the shape of the failure surface is sagging in nature. The detailed parametric study shows that the results are comparable to other suitable methods established earlier. The present study shows that the seismic passive earth pressure co- 
efficient $\left(\mathrm{K}_{\mathrm{p}}\right)$ increases due to the increase in wall friction angle $(\delta)$, soil friction angle $(\Phi)$, cohesion (c) and adhesion $\left(\mathrm{c}_{\mathrm{a}}\right)$; at the same time the value of $\mathrm{K}_{\mathrm{p}}$ decreases with the increase in wall inclinations $(\alpha)$, wall height $(\mathrm{H})$, and seismic accelerations $\left(\mathrm{k}_{\mathrm{h}}, \mathrm{k}_{\mathrm{v}}\right)$. The inclination of the failure surface with vertical increases with the decrease in wall inclination $(\alpha)$, wall friction angle $(\delta)$, soil friction angle $(\Phi)$ and height of retaining wall $(\mathrm{H})$. Whereas, the inclination of the failure surface with vertical decreases with the increase in wall inclination $(\alpha)$, soil friction angle $(\Phi)$, cohesion (c), adhesion $\left(\mathrm{c}_{\mathrm{a}}\right)$ and wall friction angle $(\delta)$. The generation of sagging nature of rupture surface (in passive condition) is showing curvilinear response in the determination passive earth pressure coefficient acting on the retaining walls.

\section{NOTATIONS}

$\Phi=$ Soil friction angle.

$\delta=$ Wall friction angle.

$\alpha=$ Wall inclination angle with the vertical.

$\mathrm{P}_{\mathrm{p}}=$ Passive earth pressure.

$\mathrm{k}_{\mathrm{h}}=$ Horizontal seismic co-efficient.

$\mathrm{k}_{\mathrm{v}}=$ Vertical seismic co-efficient.

$\mathrm{R}=$ Soil reaction force.

$\gamma=$ unit weight of soil.

$\mathrm{K}_{\mathrm{p}}=$ Passive earth pressure coefficient

$\mathrm{c}=$ cohesion

$\mathrm{c}_{\mathrm{a}}=$ adhesion

\section{REFERENCES:}

[1]. Azad, A, Shahab Yasrobi, S, Pak, A. (2008), "Active Pressure Distribution History Behind Rigid Retaining Walls", Soil Dynamics and Earthquake Engineering 28 (2008) 365-375.

[2]. Choudhury, D and Nimbalkar, S, (2005), "Seismic Passive Resistance by Pseudo-dynamic Method", Geotechnique; 55(9): 699-702.

[3]. Coulomb, C.A. (1776), "Essai Sur Une Application Des Maximis et Minimis a Queques problems Des Statique Relatifsal'Architecture", Nem. Div. Sav.Acad, Sci.Vol.7.

[4]. Ghanbari, A and Ahmadabadi, M. (2010), "PseudoDynamic Active Earth Pressure Analysis of Inclined Retaining Walls Using Horizontal Slices Method", Transaction A: Civil Engineering Vol. 17, No. 2, pp. 118-130@ Sharif University of Technology.

[5]. Ghosh, S. and Sengupta, S. (2012)," Formulation of Passive resistance on non vertical retaining wall backfilled with c- $\Phi$ ", Civil and Environmental Research ISSN 2224-5790 (Print) ISSN 2225-0514 (Online), Vol 2, No.1, 2012.

[6]. Ghosh, S. and Sharma, R. P. (2012), "Pseudo-Dynamic Evaluation of Passive Response on the Back of A Retaining Wall Supporting c- $\Phi$ Backfill”, Geomechanics and Geoengineering: An International Journal, Vol. 7, No. 2, June 2012, 115-121.
[7]. Kumar J, Subba Rao K. S, (1997), "Passive Pressure determination by method of Slices", Int J Num Anal Method Geomech USA 21:337-345.

[8]. Kumar, J. (2001), "Seismic Passive Earth Pressure Coefficients for Sands", Can. Geotech. J., Ottawa, 38, pp: 876-881.

[9]. Mononobe, N. and Matsuo, H. (1929), "On the Determination of Earth Pressure During Earthquakes" Proc. of the World Engineering Congress, Tokyo, 9, pp. 179-87.

[10]. Okabe, S. (1926), "General Theory of Earth Pressure", J. Japan Soc. Civil Eng., 12(1).

[11]. Rankine, W. J. M. (1857), "On the Stability of Loose Earth", Phil. Tras. Royal Society (London).

[12]. Shukla, S. K and Habibi, D. (2011), "Dynamic Passive Pressure from c- $\Phi$ Soil Backfills", Soil Dynamics and Earthquake Engineering 31 (2011) 845-848.

[13]. Subbarao, K. S, and Choudhury, D. (2005), "Seismic Passive Earth Pressure in Soils", Journal of Geotechnical and Geoenvironmental Engineering, ASCE 2005; 131(1); $131-5$.

[14]. Terzaghi, K, (1943), “Theoretical Soil Mechanics": John Wiley \& Sons, New York, $510 \mathrm{p}$.

\section{BIOGRAPHIES:}

Sima Ghosh presently working as an Assistant Professor at

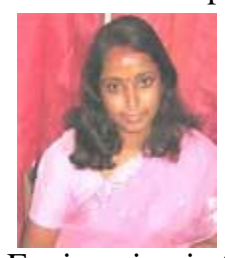
NIT Agartala, INDIA is a consistent Gold medalist in BE (Civil) from Tripura Engineering College (now NIT Agartala) in 1994 and $M$. Tech in Geotechnical Engineering from IIT, Roorkee in 2001. She has completed her $\mathrm{PhD}$ in Geotechnical Engineering in 2012 and became the very first person to attain the $\mathrm{PhD}$ Degree since the inception of $\mathrm{PhD}$ courses at NIT Agatala. She has a wide range of publications in worldwide International and National Journals including ASCE, SPRINGER, ELSEVIER, TAYLOR \& FRANCIS, EJGE, IGJ etc. Her present research works include seismic response of retaining walls, earthquake engineering, seismic bearing capacity and microzonation.

Sumen Deb is a final year M. Tech student of Geotechnical

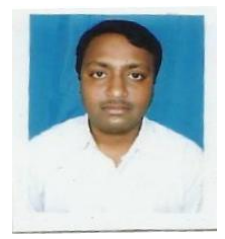
Engineering at NIT Agartala doing thesis work on Seismic response of retaining wall and waterfront retaining walls. Apart from this, he is an Engineer (Civil) serving under Rural Development Department of Government of Tripura, INDIA for the last 10 (ten) years. 